\title{
Mapping the mental space of emotional concepts through kinematic measures of decision uncertainty
}

Laura Barca ${ }^{1}$, Matteo Candidi 2, Gian Luca Lancia ${ }^{1}$, Valerio Maglianella 2, Giovanni Pezzulo $^{1}$

${ }^{1}$ Institute of Cognitive Sciences and Technologies, National Research Council, Rome, Italy ${ }^{2}$ University of Rome 'La Sapienza', Rome, Italy

Conflict of Interest Statement. The authors declare that the research was conducted in the absence of any commercial or financial relationships that could be construed as a potential conflict of interest.

Corresponding author:

Laura Barca - laura.barca@istc.cnr.it

\section{ISTC-CNR}

Via San Martino della Battaglia 44,

00185 Rome, Itlay

+39644595312

ORCID ID: $h$ ttps://orcid.org/0000-0002-8553-7717 


\begin{abstract}
Emotional concepts and their mental representations have been extensively studied. Yet, some ecologically relevant aspects, such as how they are processed in ambiguous contexts (e.g., in relation to other emotional stimuli that share similar characteristics), are incompletely known. We employed a similarity judgment of emotional concepts and manipulated the contextual congruency of the responses along the two main affective dimensions of hedonic valence and physiological activation, respectively. Behavioural and kinematics (mouse-tracking) measures were combined to gather a novel 'similarity index' between emotional concepts, to derive topographical maps of their mental representations. Self-report (interoceptive sensibility, positive-negative affectivity, depression) and physiological measures (heart rate variability) have been collected to explore their possible association with emotional conceptual representation. Results indicate that emotional concepts tipically associated to low arousal profit by contextual congruency, with faster responses and reduced uncertainty when contextual ambiguity decreases. The emotional maps recreate two almost orthogonal axes of valence and arousal, and the similarity measure captures the smooth boundaries between emotions. The emotional map of a subgroup of individuals with low positive affectivity reveals a narrower conceptual distribution, with variations in positive emotions and in those with reduced arousal (as individuals with reduced heart rate variability). Our work introduces a novel methodology to study emotional conceptual representations, bringing the behavioral dynamics of decision-making processes and choice uncertainty into the affective domain.
\end{abstract}

Keywords: emotional concepts; interoception; mouse tracker; similarity judgement task; decision uncertainty; metal map 


\section{Introduction}

During our lives, we all experience a range of affective and emotional states, but how these are mentally organized remains controversial. Despite the undeniable sensory and visceral origin of emotions, the idea that emotions are "natural kinds" and that we have pre-wired, dedicated neural circuits to map sensory and interoceptive streams into specific emotion categories is inconsistent with recent empirical data [1]. This suggests that affective and emotional states may result from a constructive process [2] that depends on contextual conditions and links perception and bodily states leading to the establishment of emotion categories and possibly organizes them into a "mental map", within which emotional concepts that have similar (different) dimensions are closer (farther).

Previous research has stressed that two pivotal affective dimensions - namely, hedonic valence and physiological activation / arousal - may contribute in organizing mental maps of emotions. For example, the prominent circumplex model of affect proposes that all affective states arise from two independent neurophysiological systems that code valence (spanning a pleasure-displeasure or positive-negative continuum) and arousal (reflecting the level of physiological activation), respectively [3-5]. The importance of these two dimensions, and especially of physiological factors, emerges from embodied accounts of emotion, too, which propose that emotional concepts are grounded in the bodily sensations (i.e., physiological, motor, and sensory streams) that characterized our prior affective experiences [6,7]. In particular, interoceptive and visceral information from our internal organs are linked to brain functions via multiple pathways and play a key role in determining our emotional experience [8-10]. Finally, interoceptive dysfunctions might concur in the development and maintenance of a variety of pathological conditions that bare clear relations with emotional states, such as eating disorders [11-14], panic disorder [15], depression [16,17] and somatic symptoms disorders [18,19] and the contribution of these physiological alterations to emotional mental representations may benefit by setting up a continuous behavioral task that measures emotion-similarity decisions.

A recent study that attempted to map the mental organization of 100 emotions and feelings derived a sophisticated topographical map, which resulted organized along the valence and arousal dimensions, along with other dimensions reflecting mental saliency. The study identified the following five clusters of emotions and feesings: negative emotions (e.g., sadness), positive emotions (e.g., pride), illness (e.g., dizziness), homeostasis (e.g., hunger) and cognition (e.g., reasoning) [20]. Topographical visualization tools efficiently convey the complexity of the feeling dimensions supporting a taxonomy of feeling concepts and words, such as compassion and awe [21,22] and modalities of expression, such human voice and prosody [23], towards a more thourough characterization of the richness of the emotional experiences.

Despite these advancements, a topic that remains largely unaddressd is the sensitivity of emotional representations to the context in which they are processed. The above methods focus on a unique, context-independent map of emotional concepts. However, emotional concepts are not processed in the void, but rather in the context of other emotional stimuli 
and more broadly of a variety of factors (e.g., emotional dispositions, physiological or interoceptive states) that are known to impact emotion perception and representation [810,24]. Such contextual factors could influence emotional processing, perhaps in axisspecific ways. For example, physiological factors might influence more significantly the arousal dimension compared to the valence dimension.

In this study, we aimed to shed light on the behavioral readouts of emotional associations and their integration into a mental map, by addressing two main sets of questions. The first set of questions regards whether we associate emotional concepts that (are typically rated to) vary along valence and arousal dimensions in distinct ways; whether these associations vary within congruent or incongruent arousal/valence contexts (e.g., when a stimulus with high arousal is processed in the presence of stimuli with high versus low arousal) and as a function of physiological factors, such as individuals' Heart Rate Variability (HRV). The second set of questions regards how we organize emotional concepts that vary along valence and arousal axes into a coherent emotional mental map and in which ways this mental organization changes as a function of individual's differences in interoception abilities and emotional dispositions (interoceptive sensibility, interoceptive awareness, affectivity orientation, depressive mood) and of physiological factors (HRV).

To address these questions, we designed an experiment that systematically manipulates the context in which emotional stimuli need to be associated with one out two options. The two options to which a target stimulus had to be associated varied for hedonic valence and physiological arousal (e.g., brave, happy and anxious) and servered as stimuli in a similarity judgment task. One stimulus (target) was centrally presented, whereas the other two were presented at the top-left and top-right corners of the screen. Participants had to respond by moving the computer mouse and click on the stimulus more similar to the centrally presented (target) stimulus. The similarity judgment task was implemented using the mouse tracker software [25], which tracks movement kinematics during the choice that are revelatory of response uncertainty and changes of mind during the decision [26,27]. Crucially, all the stimuli varied along the two key dimensions of valence and arousal, which that have been most consistently identified across various studies [3-5] and which have the higher explanatory power of the semantic structures of emotional concepts across different languages [28]. The target stimulus could be semantically congruent (i.e., have the same valence and/or arousal dimension) with one response option, both or none, therefore defining three levels of contextual ambiguity for each dimension.

To explore a possible relation between personality factors and visceral/autonomic processes and the processing and conceptual organization of emotions, we collected individuals' self-reported interoceptive and emotion measures (i.e., interoceptive sensibility, interoceptive awareness, affectivity orientation, depressive mood) and a measure of resting-state Heart Rate Variability (HRV), which is a measure of the variation in the time interval (ms) between consecutive heartbeats. Ample evidence shows that interoceptive abilities impacts emotion perception and representation, as well as emotional 
disposition and the development of clinical conditions [6,14,24,29]. Beyond the affective domain, interoception has been recently linked to conceptual representations and abstract concepts [30-32]. The innovative aspect of our present study is that of testing whether these influences may affect the structure of an emotional concepts map which geometrical properties are based on behavioral measures of the decision process.

The crucial role of HRV has particularly emerged in the context of the polyvagal perspective of behavior, which links visceral signals from the autonomic nervous system (ANS) to emotion regulation and social affiliation [33]. In this perspective, a major role in conveying visceral signals is played by the vagus nerve and HRV could be an indirect measure of vagal activity and the interaction between sympathetic (SNS) and parasympathetic nervous system (PNS). Besides, HRV has been associated to many psychological processes, such as emotion regulation, attention and decision-making [34,35], which are thought to be involved in the task devised for this study.

We formulated three sets of predictions. The first set of predictions concerns participants' response behavior during the similarity judgment task. We expected the kinematic response parameters (e.g., speed and curvature of the trajectories) to reflect a graded competition between the two response options, with higher levels of uncertainty when both responses were incongruent with the target stimulus (e.g., positive valence target with two negative valence responses ), and lower uncertainty when only one response was congruent with the target stimulus (e.g., positive valence target with one negative/one positive response). The trial in which both responses were congruent with the target stimulus being considerd an intermediate case of uncertainty. Notably, the similarity (or congruency) between target and responses could arise along both valence and arousal dimensions, which permits assessing whether contextual ambiguity affects these dimensions differentially. We expect the evaluation along the valence dimension to be more categorical and the evaluation along the arousal dimension to be more sensitive to the choice context, which might be reflected in a facilitation (e.g., faster reaction time) during the response to stimuli that are congruent with the target. Finally, we expect low HRV to be reflected in cognitive rigidity [36,37] and in particular, that low individual's HRV could be associated to poor decision-making [38], which could be indexed at a kinematic level by higher decision uncertainty.

The second set of predictions concerns the organization of emotional concepts into a mental map derived by behavioral indexes. In keeping with previous evidence [3-5], we expect the emotional concepts to be organized along the two axes of valence and arousal, with 'similar' emotions such as, for example, 'terrified' and 'scared' (with high similarity according to both hedonic valence and physiological activation) to be topographically localized closer to each other compared to dissimilar concepts such as 'relaxed' and 'anxious' (which differ on both hedonic valence and physiological activation) that would be located more distant in the map. Furthermore, we expect that at the group level, the semantic organization of concepts spans gracefully the two dimensions of valence and arousal, rather than being strongly polarized. 
The third set of predictions concerns the ways individual differences in interoceptive and emotional characteristics (i.e., interoceptive sensibility, interoceptive awareness, affectivity orientation, depressive mood) and physiological variability (HRV) might influence the organization of emotional concepts into the mental map. Specifically, we hypothesize that: 1) individuals with higher negative affectivity might have a coarser mental representation of emotional concepts, which might lack the typical multidimensionality [20,39], being anchored to the valence dimension (i.e., pleasant-unpleasant axis) and less on arousal (i.e., level of physiological activation); 2) individuals with higher interoceptive sensitivity and/or awareness might have a more refined mental representation of emotional concepts along the arousal axis compared to low-sensitive/aware individuals; 3) individuals with low levels of HRV might organize emotional concepts according to a simple, dichotomic (positive/negative) axis, while disregarding more fine-grained distinctions, especially along the arousal dimension. This is because, we hypothesize, making fine-grained distinctions between emotions along the arousal dimension requires capturing subtle differences between interoceptive streams - whereas individuals with low levels of HRV might process their interoceptive streams too rigidly. To provide some initial evidence for these hypotheses, we analyse separately small subgroups of participants having emotional and physiological parameters that vary the most from the means of the whole group. Given that we recruited a limited pool of participants and that individual differences can influence the mental organization of emotional concepts in multiple ways, we consider this aspect of the research to be exploratory.

\section{Methods}

Ethical approval. The experimental procedure has been approved by the Institutional Review Board (IRB) of the Department of Psychology, Sapienza University (prot. n. 0001201, 20/07/2020).

Participants. A gender-balanced sample of 30 healthy adults (15 female), aged 21 to 37 years old (mean age 24.5 years \pm 2.7 ), took part in the study. They were right-handed and with normal or corrected to normal vision.mouse-tracking

Materials. A list of 24 emotional words have been selected from an affective norms database [40], and is listed in Appendix 1A (Table 1). Stimuli varied according to the Valence (pleasant/unpleasant), and the Arousal (high/low) dimensions and were controlled for their length in letters amd frequency of occurrence on text web (see Appendix 1A, Table 2 and 3). Positively and negatively valenced stimuli did not differ on the arousal dimension ( $\mathrm{p}>\mathrm{1}$ ), and high-low arousal stimuli did not differ on the valence dimension ( $\mathrm{p}>\mathrm{.1})$.

Procedure. The similarity judgment task was implemented using the mouse tracker software [25]. Participants had to pair emotional concepts based on their similarity, responding with the computer mouse. Triplets of emotional words that varied for Arousal (high/low - 'terrified/respectful') and Valence (pleasant/unpleasant - 'brave/angry') were presented in each trial. The 'target' concept (e.g., 'lonely') was centrally presented, and the 
two response options (e.g., 'terrified' and 'brave') were displayed in the top corners of the screen. We operationalize the "context" in which the target emotional stimulus needs to be processed as the presence of response options, which are congruent/incongruent with the two response options, along arousal and/or valence dimensions. The task was to move the mouse and click the the button once the cursor had reached the chosen option that they consider more similar to the target. No other instruction was provided to prevent response bias.

The experiment included 288 trials, divided into two blocks with a pause in between. Each trial consisted of a triplet of stimuli. The 24 stimuli have been fully rotated in the tree position (central, top left and top right) according to the valence dimension, giving rise to 6 conditions of 48 trials each. A central target with positive valence resulted, for example, in the following conditions: 1) partial congruency - one response with positive valence and one with negative valence (either to the left or to the right); 2) full congruency - both responses with positive valence; 3) no congruency - both responses with negative valence. Please note that this design also provides conditions of partial congruency, full congruency and no congruency for the arousal dimension (see Appendix 1B for details). The experimental session was preceded by a practice session of 12 trials, and was about 40 minutes long.

The experimental design thus manipulated Valence Congruency and Arousal Congruency (i.e., triplets of stimuli that belong to the same or a different end of the valence and arousal continuum) and ambiguity (i.e., it used triplets of emotional concepts that are perceived as more or less similar) while measuring decision uncertainty (see below).

To begin each trial, participants clicked on the /START/ button located at the bottomcenter of the screen. Then the three stimuli appeared and remained on the screen until participants' response and for a maximum of 2000ms. Otherwise, a /TIME OUT/ message appeared. Response Choice and Response Times (RT i.e., from when participants pressed /START/ until they reached and pressed the response button) were recorded, as the $\mathrm{x}$ and $y$ coordinates of the mouse trajectories (sampling rate of approximately $70 \mathrm{~Hz}$ ). Mousetracking allows recording and analyzing continuous measures of behavioral choice (e.g., trajectory curvature, direction change), in addition to more classical measures such as reaction times. These continuous measures can be highly informative varioous cognitive processes, such as choice uncertainty and its resolution over time and changes of mind; for reviews, see [41-43]. Moreover, in this study, we incorporated the Area under the Curve (AUC) - a mouse-tracking measure of response uncertainty and competition between response alternatives [44,45] - into the 'similarity index' between emotional concepts that we use to build 2D topographical maps (see below for more details on how the maps are built).

We collected Response Choice, Response Time (RT, i.e., from when participants pressed /START/ until they reached and pressed the response button) and the AUC measure of mouse movement trajectories (i.e., the area created between the mouse trajectory and an ideal straight line connecting the start button with the response). As mentioned above, the AUC can be considered a measure of choice uncertainty: the closer the mouse trajectory is 
to this ideal line, the smaller will be the AUC, the lower the uncertainty in the choice. Responses exceeding the $2000 \mathrm{~ms}$ deadline accounted for $3.6 \%$ of the total data. The R code 'outlierKD' [46] did not detect outliers in the overall distribution of response time.

To be analized, response trajectories were first rescaled into a standard coordinate space. Then the duration of the movements (Response Time, RT) was normalized by re-sampling the time vector into 101 time-steps using linear interpolation to allow averaging across multiple trials. Linear Mixed Effect Models [47,48] and estimations statistics [49] have been used for statistical testing of behavioral and kinematic measures. We performed separate analyses of Valence and Arousal dimensions, as for each trial, it was possible to have a conflict in each of the dimensions, or both. For both analyses, RTs and AUC were analyzed by means of LMM with Valence (positive vs negative), or Arousal (high vs low), and target-response (Valence Congruency or Arousal Congruency see below).

Before the completion of the mouse-tracking task, we collected a measure of 5-min restingstate Heart Rate Variability (HRV) recorded as beat-to-beat intervals in milliseconds with the Bodyguard 2 (Firstbeat) HR monitor [50]. HRV analyses were carried out in the time domains using Kubios HRV software [51,52]. We used a common time-domain measurement to report HRV: the root mean square of successive beat-to-beat interval differences (rMSSD), which reflects vagal regulation of HR (cardiac parasympathetic activity) and is less susceptible to respiratory influences [53,54].

After the completion of the mouse tracking task, participants were asked to fill in the selfreport questionnaires. To gathered measures of interoceptive sensibility and awareness, we used the Body Perception Questionnaire (BPQ), a self-report measure of body awareness and autonomic reactivity [55] and the MAIA-2 [56]. The Positive and Negative affectivity Scale (PANAS) [57] have been used to measure the tendency the positively or negatively evaluate life events. The Beck Depression Inventory-Second Edition (BDI-II) [58] to evaluate state depressive mood.

To construct the 2D map of emotional concepts, we computed and plotted a 'similarity index' (i.e., a measure of the similarity) between emotional words on a 2D planar space, which represents the emotional words as nodes and their distance in space as (inverse) similarity. For each trial, we computed the 'similarity index' between two pairs of words: the target $\mathrm{T}$ and response $\mathrm{A}$, the target $\mathrm{T}$ and response $\mathrm{B}$. The similarity index of $\mathrm{TA}$ and TB is calculated by combining information about the selected Response Choice, the Response Time and the AUC of the mouse trajectory. When A was the selected response, we calculated the similarity index of TA as $(S)=0.6+0.2 *(\mathrm{RT})+0.2 *$ (AUC), where RT and AUC are normalized to the $[0,1]$ range and the value 0.6 is an arbitrary weight given to the correct Response Choice. This implies that the similarity TA is greater if A is the selected response (Response Choice), if the response is selected faster (Response Time) and with smaller trajectory curvature (AUC) - hence highlighting the importance of kinematic aspects of the choice. Finally, we set that $\mathrm{TA}+\mathrm{TB}=1$ for each trial, hence we could calculate TB $=1$ - TA. As participants encountered the same couple of stimuli (e.g., TA or TB) several times during the experiments (in different positions), the final similarity index considered in the 2D map was the mean of the similarity score calculated for each trial. 
Next, we plotted the similarity measures on a 2D planar space, with emotional concepts represented as nodes, whose distance in space reflects (inverse) similarity. The KamadaKawai algorithm have been used [59], which is a force-directed graph drawing algorithm which considers edges as springs and distances as spring rest distances. The algorithm finds a stable planar configuration which we plotted in the figure.

In addition to deriving a 2D map of emotional concepts across all participants, we exploited the natural variability of participants' positive and negative affectivity (as measured by the PANAS) and heart rate variability (HRV) to test the hypothesis that maps are sensitive to individual or group differences across these dimensions. For methodological reasons, we could not build maps for individual participants (because missing items prevent us from having complete maps for several participants). To circumvent this problem, we formed "supersubjects", by aggregating data from small (3-6) groups of participants having similar measures of positive/negative affectivity and heart rate variability. Focusing on participants having very high or very low scores in these measures (instead of, for example, using a median split) permits us maximizing the differences between the groups. Please note however that the scores of our participants in these measures were in the normal range and the pool of participants is small; therefore, we cannot draw strong conclusions about individual differences. Rather, the main goal of our group-level analysis was methodological: evaluating the sensitivity of this new methodology (i.e., the construction of 2D maps of conceptual knowledge based on kinematic measurements gathered from a similarity judgement task with varied ambiguity of the response context) to the different ways participants might organize their emotional concepts.

Analysis. The 'lmerTest' package [48] was used to analyzed the data. The model included two fixed effects (the Valence of the target (positive and negative valence) and its Valence Congruency with the responses (Cong0: no congruency; Cong1: one congruent response; Cong2: two congruent responses)) and the interaction between Valence and valence Congruency. We also included as random effects the intercept per participant (accounting for their variability), the slope per participant (capturing for the possibility that the effect of the fixed factors is not the same for all participants), and the by item random intercept (accounting for the fact that some items might be more difficult than others). The MuMIn R package [60] was used to compute a PseudoR2 (see also [61]), which indicates that our statistical model explained $22 \%$ of the variance with a medium to large effect size (f2 $=.28$ ) according to Cohen's conceptualization (1992). A post-hoc power calculation test (with the pwr.f2.test function in $\mathrm{R}$, within the pwr package) showed that our sample of 30 participants is enough to have a statistical power of .63 $(\mathrm{p}<.05)$.

The output of the model is presented in Appendix 1E (see Table 1). 


\section{Results}

Response Times (RT i.e., from when participants pressed /START/ until they reached and pressed the response button) for correct Response Choices are shown in Figure 1 as raincloud plots [62].
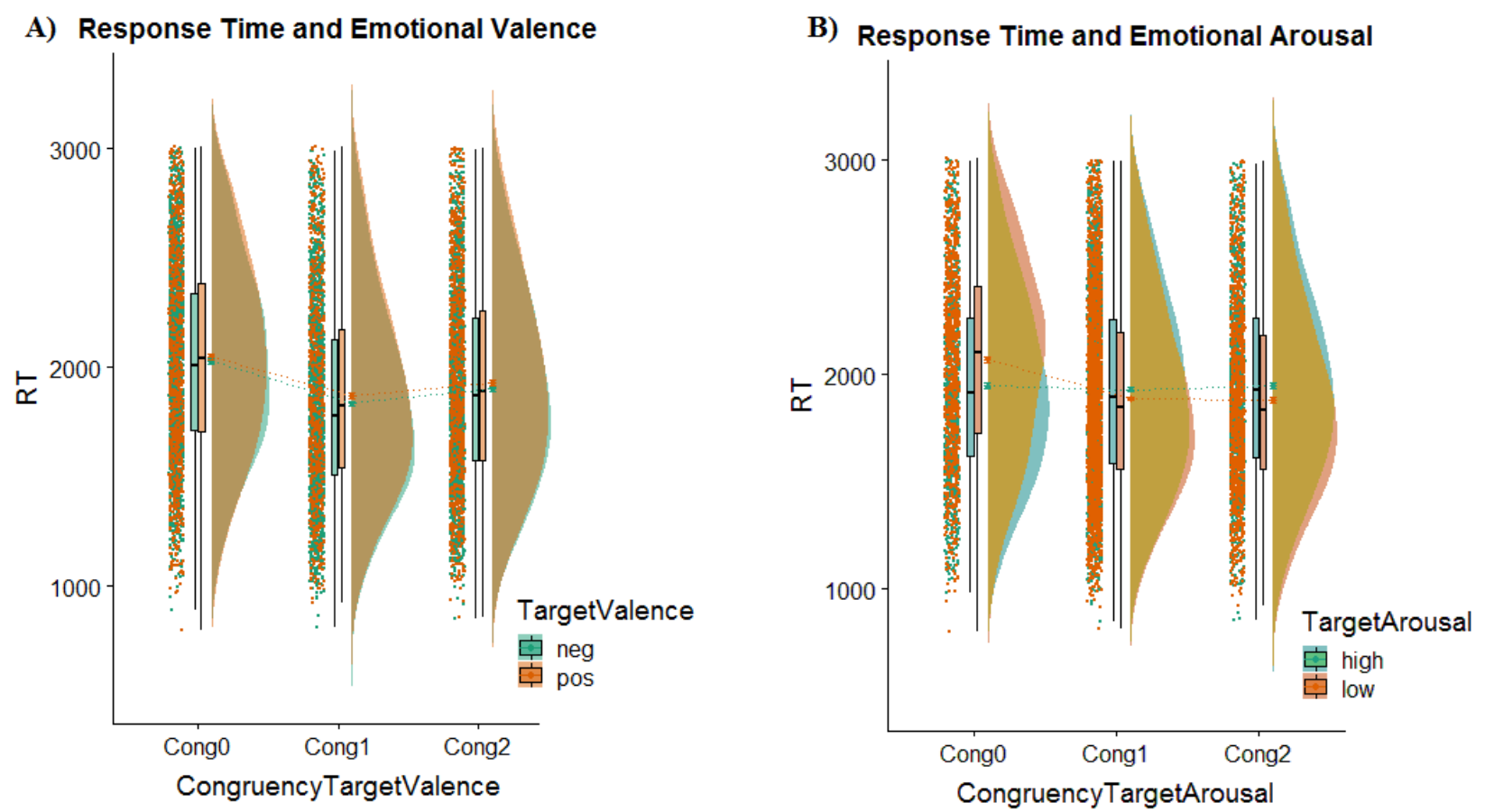

Figure 1. Raincloud plots combining a split-half violin, raw jittered data points, and a standard visualization of central tendency with boxplot of the response time for valence (Panel A) and arousal (Panel B) dimensions. RT = Resonse Time; Cong0 = no congruency between target and responses; Cong1 = target congruent with one response; Cong2 = target congruent with both responses.

\subsubsection{Analyses of targets with positive and negative valence}

The models showed that target-response Valence Congruency affected Response Times (RT i.e., from when participants pressed /START/ until they reached and pressed the response button), with faster decisions both when one response (estimate $=-195.45$, $\mathrm{p}<.001$ ), and when two responses were congruent with the target (estimate $=-130.25$, $\mathrm{p}<.001)$ compared to trials with no target-response Valence Congruency. Target's Valence did not affected RTs per se, nor it interacted with the Valence Congruency factor (ns) (see Figure 1, Panel A).

A similar pattern emerged on the Area Under the Curve (AUC) of the movement trajectory (Figure 2), which reduced in the case of one valence congruent response (estimate $=-.32, \mathrm{p}<.001$ ), and in the case of two congruent responses (estimate of -.24, $\mathrm{p}<.001)$ compared to no congruency between target and responses valence. The AUC is shown by the (average) mouse trajectories during trials with different levels of congruency in Figure 2, top panels. In Figure 2 the results are shown separatedly for targets with 
negative valence (Figure 2, top-left) and positive valence (Figure 2, top-right) for ease of comparison of our analyses of targets with high and low arousal, see below.

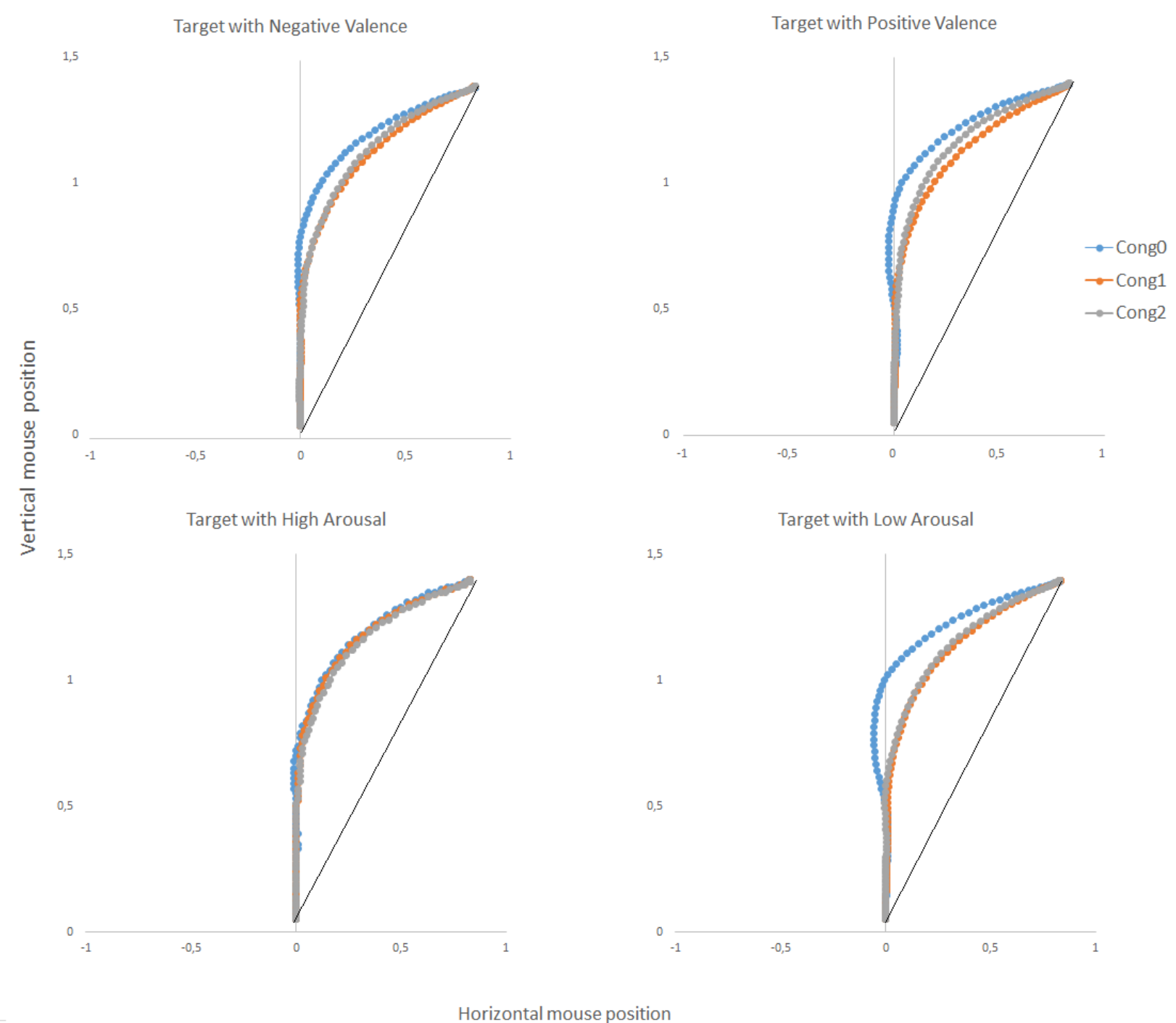

Figure 2. Mouse trajectories (averaged) as a function of valence (top panels) and arousal (bottom panels). Trajectories have been remapped to the right side, for ease of comparison. Note that the plots show that in three of the four cases (i.e, negative valence, positive valence, low arousal, but not high arousal) the Cong0 trajectories show a graded attraction towards the non-selected alternative, which is an index of greater choice uncertainty.

\subsubsection{Analyses of targets with high and low arousal}

The model included as fixed effects 1) the Arousal of the target (high and low arousal) and 2) its arousal Congruency with the responses (Cong0: no congruency; Cong1: one congruent response; Cong2: two congruent responses), and the interaction between Arousal and arousal Congruency. We included as random effects the intercept and slope 
per participant, and the by item random intercept. The output of the model is presented in Appendix 1E (see Table 2).

The model showed that variations in the target Arousal affected Response Times (RT, i.e., from when participants pressed /START/ until they reached and pressed the response button), with overall faster responses for high Arousal targets (estimate of 127.57, $\mathrm{p}<.001$ ), and interacted with response Arousal Congruency. The Arousal Congruency factor alone did not affect Response Times (ns). Multiple two-groups comparisons performed with estimation graphics (see Supplementary Figures S1 and S2) and computation of Cohen's effect size $d$ [63] showed that targets with high Arousal are not sensitive to the variation in contextual Arousal Congruency (all $d<.2$, p-values of permutation t-tests $>.1$ ). Differently, low Arousal targets were processed faster with one congruent response compared to no congruency $(\mathrm{d}=-.36, \mathrm{p}<.01)$, and when two responses were congruent compared to no congruency $(\mathrm{d}=-.48, \mathrm{p}<.001)$ (Figure 1$)$.

The movement trajectories (AUC) of targets with high Arousal (Figure 2, bottom-left panel) were not affected by the Congruency with the responses. Instead, differences according to the congruence of the context emerge in the case of targets with low Arousal (Figure 2, bottom-right panel), which appear to have a gradual attraction between the response alternatives. LMMs on AUC confirmed the effect of the Arousal fixed factor (estimate of .23, $\mathrm{p}<.001$ ) and its interaction with the Congruency factor, in case of one congruent response (estimate of -.30, p<.001) and two congruent responses (estimate of $.31, \mathrm{p}<.001)$. AUC values of target with low arousal are smaller when one $(\mathrm{d}=-1.09, \mathrm{p}<001)$ and when two $(\mathrm{d}=-1.04, \mathrm{p}<001)$ responses are cogruent respect to no congruency.

\subsection{Self-report questionnaires and heart rate variability (HRV)}

The scores of the questionnaires are reported in Appendix 1C (Table 1 reports BPQ, PANAS and BDI-II; Table 2 reports the MAIA-2 subscales). Overall, being a non-clinical population, the scores of the various questionnaires fall within the values of the respective normative samples.

\subsection{D topographical map of emotional concepts}

Next, we explored how emotional concepts were organized according to the valence and arousal dimensions by visualizing a sematic/affective mental space in a 2D topographical map; see Figure 3. Note that in Figure 3, we marked the nodes according to normative measures of arousal and valence of the affective norms database [40]. Arousal is represented as nodes size (big for high arousal, small for low arousal), while Valence as node color (blue for pleasant and red for unpleasant). 


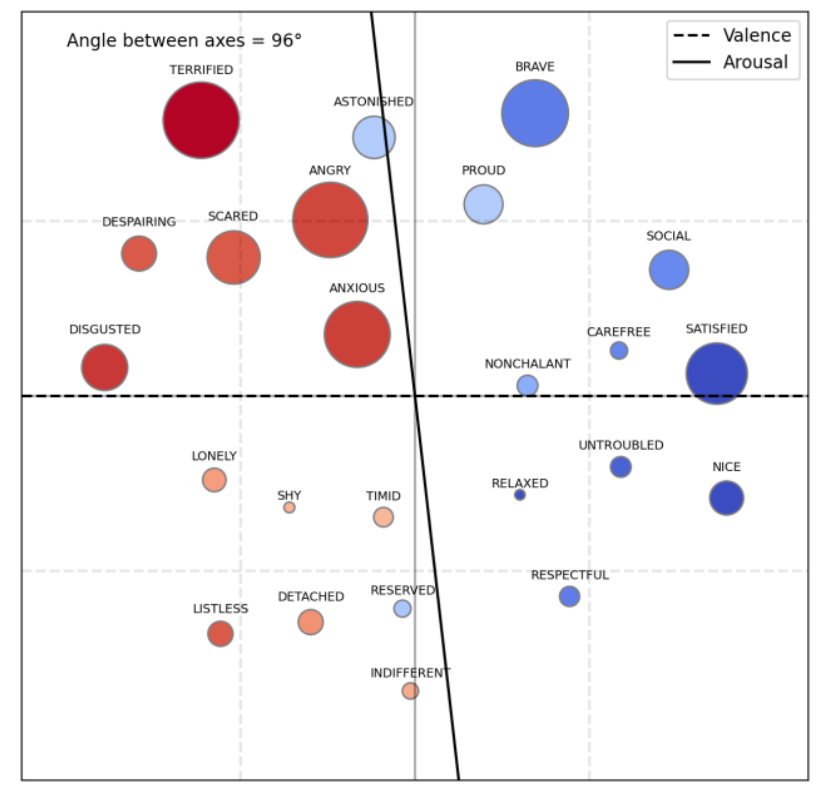

Figure 3. Two-dimensional map of emotional concepts of the whole participant group (30 participants), based on the similarity task averaged distance between stimuli. Colorcoding indicates Valence (blue: pleasant; red: unpleasant) and size of the node indicates Arousal associated to the emotion (big: high arousal; small: low arousal). Each axis is the line centered in the origin (set at the barycented of the nodes/stimuli) with an orientation that maximises the spread of the projection of the arousal (or valence) values of the stimuli on the axis (i.e., the Pearson correlation between the axis and the arousal (or valence) values of the stimuli).

The emotional concepts resulted coherently organized along the valence and arousal dimensions, and into four quadrants formed by the two almost orthogonal axes (with a $96^{\circ}$ angle between). This is noteworth, to the extent that the two axes are not assumed to be orthogonal a-priori; rather, they represent the lines with an orientation that maximises the spread of the projection of the arousal (or valence) values of the stimuli on the axis (i.e., the Pearson correlation between the axis and the arousal (or valence) values of the stimlui). Overall, the concepts resulted correctly allocated along the two dimesions with only few exceptions, or misclassifications (3 out of 24 for arousal, and 2 out of 24 for valence). We define misclassifications as cases in which emotional concepts appear in the 2D map outside the "quadrant" in which they should be (e.g., top-left quadrant of Figure 3 for unpleasant, high arousal concepts). Such misclasifications suggest that participants might have used other (unknown) dimensions in addition to the two axes of valence and arousal considered here, but the impact of these additional dimensions is minimal. Valence and arousal are confirmed to be critical dimensions to the conceptual organization of emotional concepts, especially considering that the similarity judgments from which the metrics for the maps were derived were implicit judgments: participants were not explicitly asked to process the stimuli according to their valence/arousal dimensions. 
In sum, our results suggest that in the mental map of "average" subjects, emotional concepts are organized along two near-orthogonal dimensions of valence and arousal.

Crucially, the map shown in the Figure 3 might serve as a reference map for comparison with the mental representation of emotional concepts of other population groups.

In the following sections, we test the sensitivity of our methodology in capturing differences in the organization of the emotional concepts of small subgroups of participants, i.e., "supersubjects" that differ for affective or personality factors.

\subsubsection{Map of emotional concepts in subgroups characterized by Low Positive and High Negative affectivity}

Our sample of participants were found to be within the normal range for the personality and interoceptive measures that we considered.

Among the self-report measures considered, the PANAS offers greater variability between subjects, thus we devided the sample according to the positive and negative affectivity socres.

Bearing in mind that splitting a normal sample is always a questionable artifice, we chose the criterion of selecting and grouping participants with scores 1 standard deviation above or below the mean of the normative sample. Specifically, we identified a group of 6 participants with a low level of positive affect, with individual scores 1 standard deviation below the mean of the normative data (score 15-18), to form the Low Positivity group. Similarly, we identified 6 participants with a high level of negative affect, with individual scores 1 standard deviation above the mean of the normative data (score ranging from 23 to 26), to form the High Negativity group. As explained above, these (small) groups could be equivalently considered as "supersubjects", which help us testing the sensitivity of this methodology to the different ways our participants may organize their emotional concepts.

Both positive (i.e., low level of positive affect) and negative (i.e., high level of negative affect) emotional disturbances have been associated with depression and other psychopathological conditions [64,65]. Although the scores of the participants we identified here are not in the pathological range, we tested the hypothesis that the mental maps of emotional concepts of the two subgroups was different compared to the map gathered by the whole participant group. 

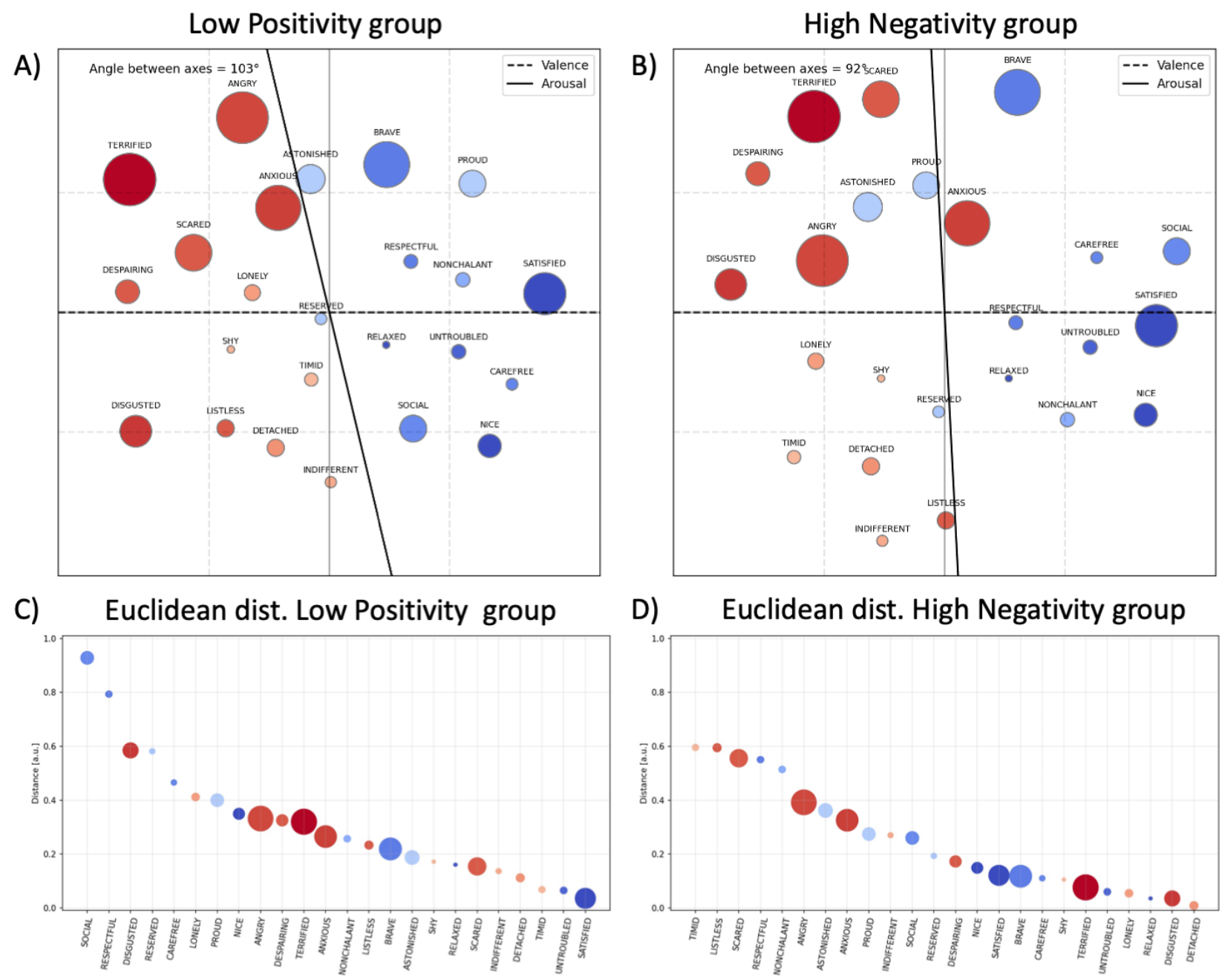

E)
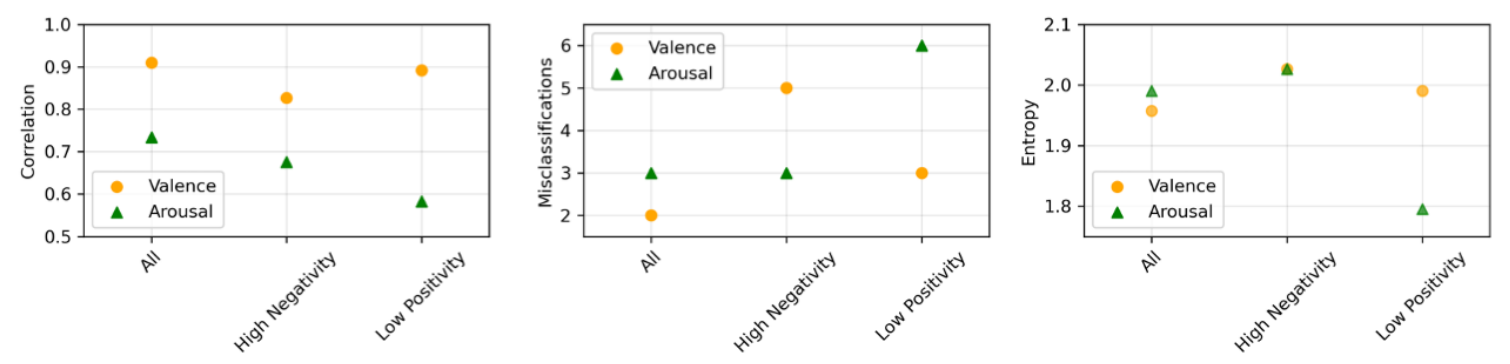

Figure 4. Results for the Low Positivity and High Negativity groups. Panel A-B: Twodimensional map of the emotional space based on the similarity task averaged distance between stimuli for the (A) Low Positivity group, and (B) High Negativity group. Panel CD: Distance between the location of each emotional concept in the map of the whole participant group and the map of the (C) Low Positivity (i.e., the euclidean distance of each item in the valence-arousal plane of the two groups) and (D) High Negativity group. Panel E: Pearson correlations between the scores of valence or arousal and the projections on the two respective axes. This and the following indexes are calculated for the maps of different participant groups: All participants, participants with High Negativity, Low positivity. A higher correlation indicates that emotional concepts are better aligned along 
the axis. Misclassification of emotional concepts for the different participant groups. Entropy of the concepts across the valence and arousal dimensions. Entropy is calculated as the spread of concepts, separatedly for each axis and is an index representing whether nodes span a large or small portion of the dimension.

Figure 4 (A) shows the map of emotional concepts of participants in the Low Positivity subgroup. Despite in this subgroup the emotional concepts are coherently organized according to the valence and arousal dimensions, several concepts are placed differently compared to the map of the whole participant group. The differences between the two maps are shown more clearly in Panel C. The comparison between the maps of the whole participant group and the Low Positivity group reveals four main things. First, the angle between the two axes of valence and arousal is greater $\left(103^{\circ}\right)$ in the Low Positivity group compared to the whole participant group $\left(96^{\circ}\right)$, which implies that in the former case the two axes are less orthogonal (Figure $4 \mathrm{~A}$ ). Second, the correlation between the score of arousal and the projection on the arousal axis decreases, indicating less consistent organization along this dimension (Panel C). Third, the misclassification of emotional concepts increases, especially along the arousal dimension (Panel E). Fourth, the map of the Low Positivity group is less entropic (i.e., nodes span a smaller portion of the space) along the arousal dimension with respect to the whole participant group (Panel E).

Panel B shows the map of emotional concepts of participants in the High Negativity group. The comparison between the maps of the whole participant group and the High Negativity group reveals four main things. First, the angle between the two axes of valence and arousal slightly decreases in the High Negativity group $\left(93^{\circ}\right)$ compared to the whole participant group $\left(96^{\circ}\right)$. Second, the correlation between the scores of valence and arousal and the projection on the respective axes slightly decreases (Panel E). Third, the misclassification of emotional concepts increases, especially along the valence dimension (Panel E). Fourth, the entropy of the map of the High Negativity group is almost the same as the whole participant group (Panel E).

Thus, the overall general organization of the emotional concepts of the positive/negative affectivty subgroups was coherent with that of the full sample of participants. Notably, despite some methodological limitations (e.g., the affectivity scores within the normal range, the limited size of the groups), it was possible to appreciate some differences in the metrics of the different maps (e.g., the entropy) and in the placement of individual emotional concepts. Along this same line, below we explore the mental organization of emotional concepts according to the physiological variable considered in the study.

3.3.2 Map of emotional concepts in subgroups characterized by low or high levels of HRV (or RMSSD)

Our sample of participants were found to be within the normal range for HRV (indexed by RMSSD). However, we formed two subgroups of participants with a low level of RMSSD and high level of RMSSD, with individual scores 1 standard deviation below the mean (3 
participants) or above the mean (4 participants) of the normative data of RMSSD, respectively. We tested the hypothesis that the mental maps of emotional concepts of the two subgroups was different compared to the map gathered by the whole participant group.

Figure 5 shows the mental maps of emotional concepts of the groups with a low level of RMSSD (Panels A, C, E) and high level of RMSSD (Panels B, D, E). Overall, the comparison between the mental maps of these two groups with the whole participant group shows minor differences. However, the group with low level of $H R V$ shows an increased misclassification of arousal stimuli and a decreased misclassification of valence stimuli (Panel E). This is coherent with the possibility that low heart rate variability might make more salient a simpler dichotomic distinction into positive/negative emotions, while disregarding more fine-grained distinctions based on arousal that are more based in interoceptive streams. Finally, the group with high level of RMSSD shows a slightly increased entropy in the mental mapping of emotions. 

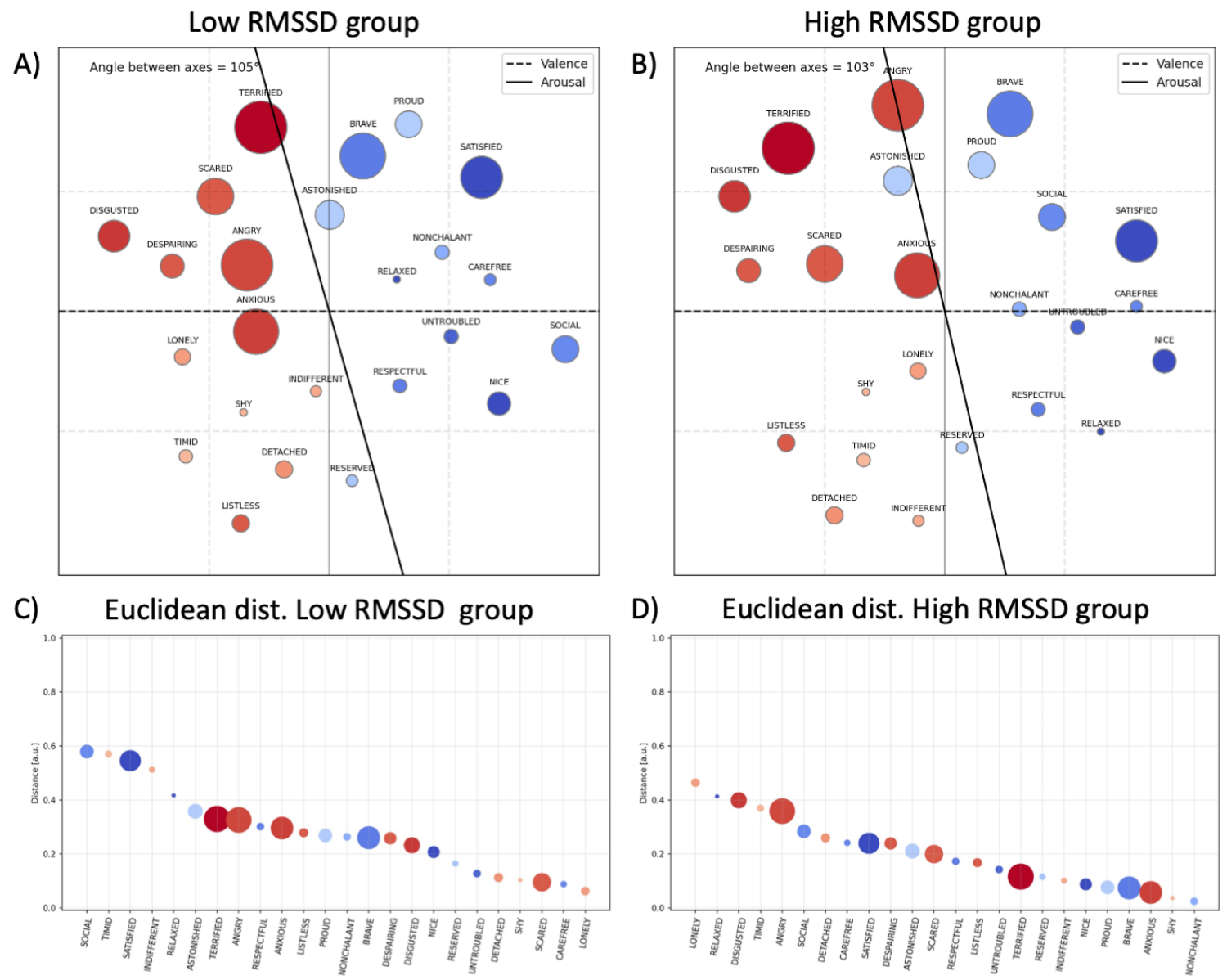

E)
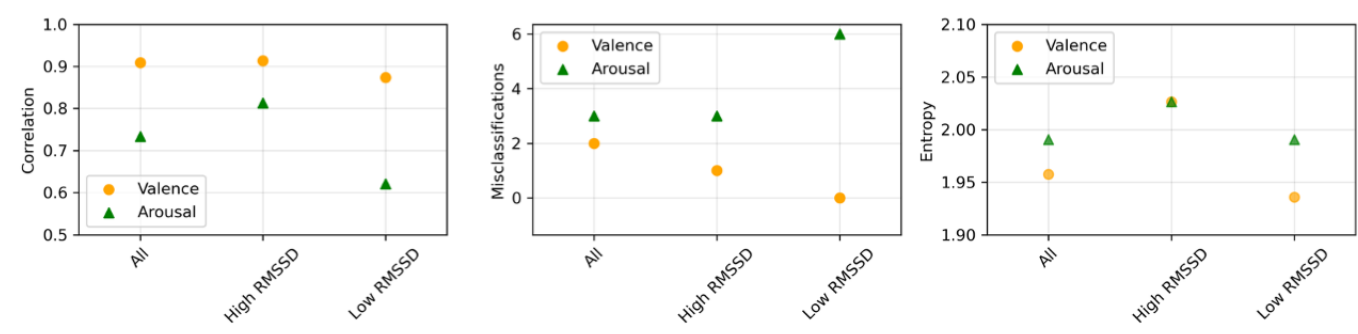

Figure 5. Mental maps of emotional concepts of participants with different levels of HRV / RMSSD. Panel A-B: Two-dimensional map of the emotional space based on the similarity task averaged distance between stimuli for the (A) Low RMSSD, and (B) for High RMSSD. Panel C-D: Distance between the location of each emotional concept in the map of the whole participant group and the map of the (C) Low RMSSD and (D) High RMSSD group. Panel E: Pearson correlations between the scores of valence or arousal and the projections on the two respective axes. This and the following indexes are calculated for the maps of different participant groups: All participants, participants with Low RMSSD, High RMSSD. Misclassification of emotional concepts for the different participant groups. Entropy of the concepts across the valence and arousal dimensions. 


\section{Discussion}

We presented a novel methodology to delve into the conceptual organization of emotional concepts. We capitalized on a longstading tradition in the study of emotional concepts based on similarity judgement tasks and topographical organization $[4,5]$. We extended these methods by incorporating novel factors, such as the ambiguity of the choice process, into the affective domain; and by assessing how physiological and affectivity variables might influence the conceptual organzation of emotions.

Our methodology derives and plots a 2-D map of emotional concept, based on behavioral and kinematic variables collected while participants use a computer mouse to classify a set of emotional concepts as a function of their (dis)similarity. The methodology also derives quantitative indexes of the mental mapping of emotional concepts, which quantify the orthogonality of the valence/arousal axes, the number (and identity) of misclassified items in the two axes and their "spread" or entropy in the map. These quantitative indexes can be used to perform various analyses aimed to assess the mental organization of emotional concepts in different individual or groups. To demonstrate this, we conducted a preliminary analysis aiming to assess to what extent the map is influenced by (or sensitive to) individual differences in affectivity (positive/negative) and physiological (HRV) indexs. Our preliminary results reveal that contextual ambiguity and physiological factors affect emotional processing and that differences in positive and negative affectivity reflect in the mental organization of emotional concepts.

\subsection{Processing of emotional stimuli and the effects of contextual ambiguity}

Our first research question regards the ways we process emotional stimuli that vary along valence and arousal dimensions; and to what extent this processing is influenced by contextual ambiguity - and the presence of congruent or incongruent emotional stimuli. Our analysis of behavioral and kinematic responses reveals that participants processed stimuli varied according to valence and arousal dimensions differently in our task. The hedonic valence (per se or in interaction with the congruency) did not affect the speed and trajectory curvature of the responses, wherease targets with high-arousal had an overall advantage over low-arousal targets, both in speed and trajectory's curvature. Notably, the arousal dimension interacted with the congruency factor, with low arousal stimuli processed faster and with straighter trajectories as the congruence with the responses increased. Taken together, these results suggest that the arousal dimension affects behavioral association of emotion in a more context-sensitive manner. We speculate that this reflects the notion that the processing of high arousal states might engage visceral and interoceptive information, which according to embodied theories of emotion are part and parcel of the emotional experience [8-10]. Rather, during the processing of low arousal states, where plausibly interoceptive information is less salient, participants could have engaged more categorical processing to solve the task. 
Our analyses reveal also that the congruency between the target and the responses modulates similarity judgments; and this happens for emotional stimuli that varied across both valence and arousal axes. As expected from previous studies of movement kinematics during choices [27,44], faster responses and smaller trajectory curvature emerged in conditions of low choice uncertainty, namely, when only one response was congruent with the target, along either valence or arousal axes. Slower responses and greater trajectory curvature occurred when both responses were congruent with the target or (to a larger extent) incongruent with the target, suggesting a graded competition between response alternatives during the decision process and increased choice uncertainty. The comparison between the cases when both responses were incongruent vs. congruent with the target is instructive, as it shows that when the competition remains within the same dimension (e.g., between positive-valence or high-arousal stimuli), the conflict between the choice alternatives is significantly reduced. This finding strongly suggest that participants formed categorically different representations of emotional stimuli across both valence and arousal axes.

These data also contribute to the debate on the grounding of the different types of concepts (e.g., concrete, abstract and emotional concepts). Numerous studies have shown that conceptual categories do not have clear-cut boundaries and are not homogeneous [6669]. The category of abstract concepts, for example, encompasses rather heterogeneous entities - from philosophical and spiritual concepts (e.g., 'value'), time and quantity concepts (e.g., 'beginning') to emotional and mental state concepts (e.g., 'shame') - which are grounded to various degree in sensorimotor and interoceptive experiences [32]. Future studies might employ the methodology we have described - which involves the use of kinematic measures to derive a $2 \mathrm{~d}$ map of conceptual knowledge - to characterize other dimensions of the representation of concepts in addition to the affective one (e.g., abstractness vs concreteness, exteroceptive vs interoceptive).

A novelty of our study is the use of the mouse tracker in the similiarity judgment of emotional concepts. Brooks and Freeman [70] recently used the mouse-tracking technique to study how conceptual knowledge affects emotions perception, and specifically the perception of faces expressing different emotions. Conceptual knowledge of emotions has been measured via similarity ratings of pairs of the 6 basic emotion (e.g., 'From $1=$ not at all to 10 = extremely, how similar do you find the emotions Anger and Fear?'). In the mouse-tracking task, participants had to indicate whether the face displayed had, for example, an expression of Anger or Fear. Results show that the more the emotions were rated as similar to each other, the greater the attraction exerted by the competing category on the response trajectory. Here, differently from Brooks and Freeman [70], the similarty judgments have been implemented within the mouse tracker and used triplets of emotional concepts, allowing to vary the ambiguity of the response context.

\subsection{Organization of emotional concepts in a mental map}

Our second set of research questions regards the way we organize emotional concepts into a coherent mental map and the potential differences between the maps of the whole 
participant group and of two subgroups characterized by (subclinical levels of) Low Positive affectivity and High Negative affectivity, as assessed using the PANAS. For this, we used the similarity judgements to derive a distance between emotional concepts, which permits mapping them into a two-dimentional space.

Our analysis of the 2D metric organization of emotional space shows that the two axes of valence and arousal identified in previous research are almost orthogonal, in keeping with the claim of the circumplex model that they might arise from two independent neurophysiological systems [3-5]. Furthermore, our results show that even when the topography of the map is based on behavioral indexes of emotion similarity, emotional concepts are gracefully distributed along a continuum spanning both valence and arousal dimensions, rather than being polarized, suggesting that people might be sensitive to finegrained differences between them. As proposed by [21], the fact that some emotions are close to each other suggests that there may be an easier transition between them (as in the case of 'scared' and 'angry' of our current results) or the possibility to experience different and (apparently) divergent emotions at the same time (as 'scared' and 'brave'). The existence of 'mixed emotions,' such as feeling happy and sad at the same time, might occur in typical situations [71] and is considered an indicator of greater emotional complexity and more adaptive functioning [72]; furthermore, it has been recently related with an increase in virus-preventing behaviours (e.g., social distancing) during Covid-19 pandemic [73]. However, the research on mixed emotions is extensive and so far provided conflicting results, which might be due to the definition of the phenomenon under study or to the different methods designed for eliciting and measuring mixed emotions see [74] for a discussion). Here, we simply observe that the methodology we have developed allows us to appreciate an overall view of the conceptual organization of the emotional space of the participants, and the smooth boundaries between positive and negative affect, as those with varying degrees of physiological activation.

Recent research reveals that valence and arousal properties of emotional concepts are not stable but can vary in context-sensitive ways [75-78], reflecting a constructive process [6]. Our task implicitly requires processing emotional concepts in the context of other (congruent or incongruent) concepts. Therefore, the mental maps that emerge from our analysis are likely to reflect the average of several context-sensitive judgements (or our categorical thinking about emotions [79]), rather than a "correct", context-independent value for valence and arousal properties. The sensitivity of the maps to personality and interoceptive dimensions indicates that these dimensions can provide an additional context to emotional processing that can "morph" the mental space of emotional concepts.

In some conditions, quickly shifting from one emotional state to another, or experiencing ambivalent emotions simultaneously can be unpleasant [80] and related to psychological distress [81]. The exploration of the conceptual organization of emotions, thus, might be a valid tool in clinical settings, by delving into the patients' affective domain and providing a graphic visualization of the organization of emotional concepts that is easy to understand and therefore highly usable, akin to other visualization tools more related to the bodily representations of emotions [82,83]. Furthermore, the maps described here 
permit deriving quantitative indexes (e.g., correlations between valence and arousal axes, misclassification and entropy of items) that can help shed light on the specific organization of emotional concepts of different participants or groups.

As a first step in this direction, we explored the sensitivity of our methodology in capturing individual differences in the mental organization of emotional concepts. We found differences in the maps of "supersubjects" (i.e., small subgroups formed by participants with Low Positivity, High Negativity, Low HRV and High HRV). The maps of the group Low Positive affectivity showed three convergent indexes of reduced processing of the arousal dimension: a reduced correlation between the scores of emotional concepts and their projections on the arousal index, a narrower distribution of emotional concepts along the arousal index and an increased misclassification of arousal items. In other words, participants in the Low Positivity group organized stimuli that varied along the arousal dimension in a way that is less precise and differentiated compared to the whole participant group. These findings suggest that participants in the Low Positivity group could rely less on physiological and bodily information when making emotional judgements. This is consistent with evidence that reduced positive affect is strongly associated with the tendency of suppressing emotion [84] as well as with depression and anxiety (see [65] for a review). Low positive emotionality (i.e., anhedonia) is consistently related to depression and (social) anxiety, too, althought to a weaker extent. The maps of the High Negativity group showed a different pattern, with a greater misclassification of the valence dimension. Finally, the maps of the group with Low HRV shows a similar misclassification of stimuli that varied for the arousal dimensions as the group with Low Positivity (along with a better classification of stimuli that varied for the valence dimension). This result suggests that Low HRV might rely less on interoceptive information and instead adopt a more categorical strategy to solve the task.

In sum, our results suggests that the organization of emotional concepts along the valence dimension is more stable while that of arousal varies to a greater extent based on the characteristics of the subgroups considered (and the greater/reduced reference to bodily information). While the limited pool of participants does not allow us to draw strong conclusions at the level of individuals or small groups, the above results indicate that the methodology developed here to build mental maps of emotional concepts is at least suitable to address these research questions.

\section{3 Limitations of the current study and future directions}

A limitation of the current study is that it only considered a limited number of emotional concepts compared to previous studies, which tested hundreds [20] or thousands [21] of emotional stimuli. On the other hand, using a short list of emotional concepts permitted us to study the similarity between emotional concepts with behavioural and kinematic measures, and to assess the time course of decision-making along valence and arousal dimensions. Furthermore, the use of kinematic measures permitted us to derive a detailed 'implicit' metric of similarity between stimuli, which considers not only the choice made 
but also decision time and the curvature of the decision trajectory. An open question for future research is extending the method to map a wider range of emotional concepts.

Another interesting application of the methods developed here is studying how our mental maps of emotions change at a fast timescale, as an effect of interoceptive dynamics and information from our bodies. Accumulating evidence supports the integration of interceptive dynamics into our emotional experience $[35,85,86]$. For example, passively listening to emotional words activate both the visceral system (i.e., an acceleration of heart rate when listening to words with positive valence and low arousal) and the somatic system (i.e., an increase in corrugator muscular activity when listeing to words with negative valence and low arousal) [87]. Various studies manipulated the physiological state of healthy participants, and tested how this affects the processing of stimuli with emotional content. In one of such studies, an increase in heart rate and body energy expenditure induced by physical exercise reduced the processing time of faces expressing fear (i.e., an emotion that has congruent physiological signatures) but not those espressing neutrality, disgust [88] or happiness [89], even when eliciting emotional content was not needed to perform the task (as participants performed a gender categorization). These studies suggest that the faster processing of fearful stimuli was due to the congruence between the physiological state of the participant and emotion semantics - and more broadly, that the interoceptive information from the body could be used within the (embodied) predictive processing of emotionally-charged faces [35,90-93]. Converging evidence for the influence of interoceptive streams in our judgements about bodily-related dimensions, such as effort, comes from a study that gave participants a false acustic feedback that simulated faster heart rate and found that participants reported that they were exerting more effort than it really was [94]. An open objective for future research consists in taking inspirations from these and other studies that manipulated interoceptive streams, to study whether this changes the ways we organize emotional concepts into mental maps. Similarly, it may be relevant to analyse the real-time changes in physiological parameters, such as HRV, associated to the decision-making process categorizing the emotional concepts. Furthermore, in addition to the transient interoceptive manipulations used in these studies, it would be interesting to study whether longer-term interoceptive dysfunctions, such as those observed in various psychopathologies [95], influence our mental maps of emotions. Conversely, different psychopathologies could be associated to different features in the map of the emotions, which, in turn and hopefully, could become a new tool to even assess and give new insights into the disorders.

Another open research question research regards how the organization of emotional concepts changes during development. Previous research has established that children initially build emotional categories using broad dimensions, such as the hedonic properties of emotional states (as pleasant-unpleasant), and progressively incorporating further facet, as the degree of physiological activation (as high - low arousal). In a freelabeling task of prototypical facial expression of emotions (i.e., happiness, sadness, anger, fear, surprise, disgust), young preschoolers extend the use of the label happiness to surprise 
and fear faces, gradually narrowing it to happy faces. A similar pattern emerged for other early-emerging categories as sadness and anger. The labels scared and surprised were produced mainly by older-preschooler and started more narrowed [96]. Such a pattern also emerged in categorizing emotional faces [97], confirming the gradual structuring of an adult-like organization of emotional concepts. However, mixed results are reported. In a habituation paradigm, two years old children do not spontaneously form superordinate categories of negatively valenced facial expressions (i.e., they perceive the perceptual difference between anger, sadness and disgust but not that they pertain to the 'negativevalence category') and do not take advantage from verbal labeling [98]. Another crucial aspect that remains to be investigated is how our mental organization of emotional concept changes as we develop linguistic abilities. Linguistic tools, such as words, aid the construction of emotional concepts by providing labels to which emotional experiences are anchored [68], initially broadly, and with subsequent refinement through development [96,97]. Our task uses lingustic stimuli to probe the mental organization of emotional concept; but using non-lingustic stimuli would allow testing mental maps of emotions across development, before and after linguistic abilities are developed.

\section{Acknowledgements}

This research received funding from the European Union's Horizon 2020 Framework Programme for Research and Innovation under the Specific Grant Agreement Nos. 785907 and 945539 (Human Brain Project SGA2 and SGA3) to GP; and the European Research Council under the Grant Agreement No. 820213 (ThinkAhead) to GP. The authors are grateful to Cristina Ottaviani for kindly lending the Bodyguard 2 (Firstbeat) heart rate monitor to us and to Giuseppina Porciello and Vanessa Era for their help in data collection. 


\section{References}

1. Barrett LF. 2006 Are Emotions Natural Kinds? Perspect Psychol Sci 1, 28-58. (doi:10.1111/j.1745-6916.2006.00003.x)

2. Barrett LF. 2017 How emotions are made: The secret life of the brain. Houghton Mifflin Harcourt.

3. Posner J, Russell JA, Peterson BS. 2005 The circumplex model of affect: An integrative approach to affective neuroscience, cognitive development, and psychopathology. Dev

Psychopathol 17, 715-734. (doi:10.1017/S0954579405050340)

4. $\quad$ Russell JA. 1980 A circumplex model of affect. Journal of Personality and Social Psychology 39, 1161-1178. (doi:10.1037/h0077714)

5. Barrett LF, Fossum T. 2001 Mental representations of affect knowledge. Cognition and Emotion 15, 333-363. (doi:10.1080/02699930125711)

6. Barrett LF. 2017 The theory of constructed emotion: an active inference account of interoception and categorization. Soc Cogn Affect Neurosci 12, 1-23. (doi:10.1093/scan/nsw154)

7. Hoemann K, Xu F, Barrett LF. 2019 Emotion words, emotion concepts, and emotional development in children: A constructionist hypothesis. Developmental Psychology 55, 1830-1849. (doi:10.1037/dev0000686)

8. Critchley HD, Harrison NA. 2013 Visceral influences on brain and behavior. Neuron 77, 624-638. (doi:10.1016/j.neuron.2013.02.008)

9. Seth AK, Friston KJ. 2016 Active interoceptive inference and the emotional brain. Phil. Trans. R. Soc. B 371, 20160007.

10. Pezzulo G, Barca L, Friston KJ. 2015 Active inference and cognitive-emotional interactions in the brain. Behavioral and Brain Sciences 38, e85.

11. Badoud D, Tsakiris M. 2017 From the body's viscera to the body's image: Is there a link between interoception and body image concerns? Neurosci Biobehav Rev 77, 237-246.

(doi:10.1016/j.neubiorev.2017.03.017)

12. Barca L, Pezzulo G. 2020 Keep your interoceptive streams under control: An active inference perspective on anorexia nervosa. Cogn Affect Behav Neurosci (doi:10.3758/s13415-02000777-6)

13. Khalsa SS, Hassanpour MS, Strober M, Craske MG, Arevian AC, Feusner JD. 2018 Interoceptive Anxiety and Body Representation in Anorexia Nervosa. Front. Psychiatry 9. (doi:10.3389/fpsyt.2018.00444)

14. Paulus MP, Feinstein JS, Khalsa SS. 2019 An Active Inference Approach to Interoceptive Psychopathology. Annu Rev Clin Psychol 15, 97-122. (doi:10.1146/annurev-clinpsy-050718095617)

15. Maisto D, Barca L, Van den Bergh O, Pezzulo G. 2021 Perception and misperception of bodily symptoms from an Active Inference perspective: Modelling the case of panic disorder. Psychological Review.

16. Barrett LF, Quigley KS, Hamilton P. 2016 An active inference theory of allostasis and interoception in depression. Phil. Trans. R. Soc. B 371, 20160011.

17. Stephan KE et al. 2016 Allostatic Self-efficacy: A Metacognitive Theory of Dyshomeostasis-Induced Fatigue and Depression. Front Hum Neurosci 10.

(doi:10.3389/fnhum.2016.00550)

18. Pezzulo G, Maisto D, Barca L, Bergh OV den. 2019 Symptom Perception From a Predictive Processing Perspective. Clinical Psychology in Europe 1(4), e35952.

(doi:10.32872/cpe.v1i4.35952)

19. Van den Bergh O, Witthöft M, Petersen S, Brown RJ. 2017 Symptoms and the body: Taking the inferential leap. Neurosci Biobehav Rev 74, 185-203. (doi:10.1016/j.neubiorev.2017.01.015)

20. Nummenmaa L, Hari R, Hietanen JK, Glerean E. 2018 Maps of subjective feelings. Proc. 
Natl. Acad. Sci. U.S.A. 115, 9198-9203. (doi:10.1073/pnas.1807390115)

21. Cowen AS, Keltner D. 2017 Self-report captures 27 distinct categories of emotion bridged by continuous gradients. Proc Natl Acad Sci U S A 114, E7900-E7909.

(doi:10.1073/pnas.1702247114)

22. Cowen AS, Keltner D. 2019 What the face displays: Mapping 28 emotions conveyed by naturalistic expression. Am Psychol (doi:10.1037/amp0000488)

23. Cowen AS, Elfenbein HA, Laukka P, Keltner D. 2019 Mapping 24 emotions conveyed by brief human vocalization. American Psychologist 74, 698-712. (doi:10.1037/amp0000399)

24. Parrinello N, Napieralski J, Gerlach AL, Pohl A. 2022 Embodied feelings-A meta-analysis on the relation of emotion intensity perception and interoceptive accuracy. Physiology \& Behavior 254, 113904. (doi:10.1016/j.physbeh.2022.113904)

25. Freeman JB, Ambady N. 2010 MouseTracker: software for studying real-time mental processing using a computer mouse-tracking method. Behav Res Methods 42, 226-241. (doi:10.3758/BRM.42.1.226)

26. Barca L, Pezzulo G. 2012 Unfolding visual lexical decision in time. PloS one 7, e35932.

27. Barca L, Pezzulo G. 2015 Tracking second thoughts: Continuous and discrete revision processes during visual lexical decision. PLoS One 10, e0116193.

28. Jackson JC, Watts J, Henry TR, List J-M, Forkel R, Mucha PJ, Greenhill SJ, Gray RD, Lindquist KA. 2019 Emotion semantics show both cultural variation and universal structure.

Science 366, 1517-1522. (doi:10.1126/science.aaw8160)

29. Nord CL, Garfinkel SN. 2022 Interoceptive pathways to understand and treat mental health conditions. Trends in Cognitive Sciences 26, 499-513. (doi:10.1016/j.tics.2022.03.004)

30. Borghi AM, Barca L, Binkofski F, Tummolini L. 2018 Abstract concepts, language and sociality: from acquisition to inner speech. Philos Trans R Soc Lond B Biol Sci 373, 20170134. (doi:10.1098/rstb.2017.0134)

31. Connell L, Lynott D, Banks B. 2018 Interoception: the forgotten modality in perceptual grounding of abstract and concrete concepts. Philos Trans R Soc Lond B Biol Sci 373, 20170143. (doi:10.1098/rstb.2017.0143)

32. Villani C, Lugli L, Liuzza MT, Nicoletti R, Borghi AM. 2021 Sensorimotor and interoceptive dimensions in concrete and abstract concepts. Journal of Memory and Language 116, 104173. (doi:10.1016/j.jml.2020.104173)

33. Porges SW. 2007 The polyvagal perspective. Biol Psychol 74, 116-143.

(doi:10.1016/j.biopsycho.2006.06.009)

34. Thayer JF, Lane RD. 2009 Claude Bernard and the heart-brain connection: further elaboration of a model of neurovisceral integration. Neurosci Biobehav Rev 33, 81-88. (doi:10.1016/j.neubiorev.2008.08.004)

35. Pezzulo G. 2013 Why do you fear the bogeyman? An embodied predictive coding model of perceptual inference. Cogn Affect Behav Neurosci (doi:10.3758/s13415-013-0227-x)

36. Hildebrandt LK, McCall C, Engen HG, Singer T. 2016 Cognitive flexibility, heart rate variability, and resilience predict fine-grained regulation of arousal during prolonged threat.

Psychophysiology 53, 880-890. (doi:10.1111/psyp.12632)

37. Grol M, De Raedt R. 2020 The link between resting heart rate variability and affective flexibility. Cogn Affect Behav Neurosci 20, 746-756. (doi:10.3758/s13415-020-00800-w)

38. Colzato LS, Jongkees BJ, de Wit M, van der Molen MJW, Steenbergen L. 2018 Variable heart rate and a flexible mind: Higher resting-state heart rate variability predicts better taskswitching. Cogn Affect Behav Neurosci 18, 730-738. (doi:10.3758/s13415-018-0600-x)

39. Cowen A, Sauter D, Tracy JL, Keltner D. 2019 Mapping the Passions: Toward a HighDimensional Taxonomy of Emotional Experience and Expression. Psychol Sci Public Interest 20, 69-90. (doi:10.1177/1529100619850176)

40. Montefinese M, Ambrosini E, Fairfield B, Mammarella N. 2014 The adaptation of the Affective Norms for English Words (ANEW) for Italian. Behavior Research Methods 46, 887-903. 
(doi:10.3758/s13428-013-0405-3)

41. Freeman JB, Dale R, Farmer TA. 2011 Hand in motion reveals mind in motion. Front Psychol 2, 59. (doi:10.3389/fpsyg.2011.00059)

42. Song J-H, Nakayama K. 2009 Hidden cognitive states revealed in choice reaching tasks. Trends Cogn Sci 13, 360-366. (doi:10.1016/j.tics.2009.04.009)

43. Spivey MJ, Dale R. 2006 Continuous Dynamics in Real-Time Cognition. Curr Dir Psychol Sci 15, 207-211. (doi:10.1111/j.1467-8721.2006.00437.x)

44. Hehman E, Stolier RM, Freeman JB. 2015 Advanced mouse-tracking analytic techniques for enhancing psychological science. Group Processes \& Intergroup Relations 18, 384-401.

45. Spivey M, Grosjean M, Knoblich G. 2005 Continuous attraction toward phonological competitors. Proceedings of the National Academy of Sciences of the USA 102, 10393-10398.

46. Dhana K. 2018 Identify, describe, plot, and remove the outliers from the dataset.

DataScience+.

47. Baayen RH, Davidson DJ, Bates MD. 2008 Mixed-effects modeling with crossed random effects for subjects and items. Journal of Memory and Language 59, 390-412.

48. Kuznetsova A, Brockhoff PB, Christensen RHB. 2017 lmerTest Package: Tests in Linear Mixed Effects Models. Journal of Statistical Software 82, 1-26. (doi:10.18637/jss.v082.i13)

49. Ho J, Tumkaya T, Aryal S, Choi H, Claridge-Chang A. 2019 Moving beyond P values: data analysis with estimation graphics. Nature Methods 16, 565-566. (doi:10.1038/s41592-019-0470-3)

50. Parak J, Tarniceriu A, Renevey P, Bertschi M, Delgado-Gonzalo R, Korhonen I. 2015 Evaluation of the beat-to-beat detection accuracy of PulseOn wearable optical heart rate monitor. Annu Int Conf IEEE Eng Med Biol Soc 2015, 8099-8102. (doi:10.1109/EMBC.2015.7320273) 51. Penttilä J, Helminen A, Jartti T, Kuusela T, Huikuri HV, Tulppo MP, Coffeng R, Scheinin H. 2001 Time domain, geometrical and frequency domain analysis of cardiac vagal outflow: effects of various respiratory patterns. Clin Physiol 21, 365-376. (doi:10.1046/j.1365-2281.2001.00337.x) 52. Task Force of the European Society of Cardiology and the North American Society of Pacing and Electrophysiology. 1996 Heart rate variability: standards of measurement, physiological interpretation and clinical use. Task Force of the European Society of Cardiology and the North American Society of Pacing and Electrophysiology. Circulation 93, 1043-1065.

53. Tarvainen MP, Niskanen J-P, Lipponen JA, Ranta-Aho PO, Karjalainen PA. 2014 Kubios HRV--heart rate variability analysis software. Comput Methods Programs Biomed 113, 210-220. (doi:10.1016/j.cmpb.2013.07.024)

54. Tarvainen MP, Ranta-Aho PO, Karjalainen PA. 2002 An advanced detrending method with application to HRV analysis. IEEE Trans Biomed Eng 49, 172-175. (doi:10.1109/10.979357)

55. Porges S. 1993 Body perception questionnaire: Laboratory of development assessment. University of Meriland.

56. Mehling WE, Acree M, Stewart A, Silas J, Jones A. 2018 The Multidimensional Assessment of Interoceptive Awareness, Version 2 (MAIA-2). PLOS ONE 13, e0208034.

(doi:10.1371/journal.pone.0208034)

57. Watson D, Clark LA, Tellegen A. 1988 Development and validation of brief measures of positive and negative affect: The PANAS scales. Journal of Personality and Social Psychology 54, 1063-1070. (doi:10.1037/0022-3514.54.6.1063)

58. Montano A, Flebus GB. 2006 Presentazione del Beck Depression Inventory- Seconda Edizione (BDI-II): conferma della struttura bifattoriale in un campione di popolazione italiana. Psicoterapia Cognitivo e Comportamentale 12, 67-82.

59. Kamada T, Kawai S, others. 1989 An algorithm for drawing general undirected graphs. Information processing letters 31, 7-15.

60. Bartoń K. 2019 MuMIn: Multi-Model Inference. See https://CRAN.Rproject.org/package=MuMIn.

61. Nakagawa S, Schielzeth H. 2013 A general and simple method for obtaining R2 from generalized linear mixed-effects models. Methods in Ecology and Evolution 4, 133-142. 
(doi:10.1111/j.2041-210x.2012.00261.x)

62. Allen M, Poggiali D, Whitaker K, Marshall TR, Kievit RA. 2019 Raincloud plots: a multiplatform tool for robust data visualization. Wellcome Open Research 4, 63.

(doi:10.12688/wellcomeopenres.15191.1)

63. Cumming G. 2013 Understanding the new statistics: Effect sizes, confidence intervals, and meta-analysis. Routledge.

64. Suslow T, Bodenschatz CM, Kersting A, Quirin M, Günther V. 2019 Implicit affectivity in clinically depressed patients during acute illness and recovery. BMC Psychiatry 19.

(doi:10.1186/s12888-019-2365-3)

65. Watson D, Naragon-Gainey K. 2010 On the specificity of positive emotional dysfunction in psychopathology: Evidence from the mood and anxiety disorders and schizophrenia/schizotypy.

Clinical Psychology Review 30, 839-848. (doi:10.1016/j.cpr.2009.11.002)

66. Mazzuca C, Barca L, Borghi AM. 2017 The peculiarity of emotional words: a grounded approach. Rivista internazionale di Filosofia e Psicologia 8, 124-133.

67. Villani C, Lugli L, Liuzza MT, Borghi AM. 2019 Varieties of abstract concepts and their multiple dimensions. Language and Cognition 11, 403-430. (doi:10.1017/langcog.2019.23)

68. Borghi AM, Barca L, Binkofski F, Castelfranchi C, Pezzulo G, Tummolini L. 2018 Words as social tools: Language, sociality and inner grounding in abstract concepts. Physics of Life Reviews (doi:10.1016/j.plrev.2018.12.001)

69. Dove G, Barca L, Tummolini L, Borghi AM. 2020 Words have a weight: language as a source of inner grounding and flexibility in abstract concepts. Psychological Research (doi:10.1007/s00426-020-01438-6)

70. Brooks JA, Freeman JB. 2018 Conceptual knowledge predicts the representational structure of facial emotion perception. Nat Hum Behav 2, 581-591. (doi:10.1038/s41562-018-0376-6)

71. Larsen JT, McGraw AP, Cacioppo JT. 2001 Can people feel happy and sad at the same time? Journal of Personality and Social Psychology 81, 684-696. (doi:10.1037/0022-

3514.81.4.684)

72. Hershfield HE, Scheibe S, Sims TL, Carstensen LL. 2013 When Feeling Bad Can Be Good: Mixed Emotions Benefit Physical Health Across Adulthood. Soc Psychol Personal Sci 4, 54-61. (doi:10.1177/1948550612444616)

73. Oh VYS, Tong EMW. 2021 Mixed Emotions, but Not Positive or Negative Emotions, Facilitate Legitimate Virus-Prevention Behaviors and Eudaimonic Outcomes in the Emergence of the COVID-19 Crisis. Affect Sci 2, 311-323. (doi:10.1007/s42761-021-00045-x)

74. Charles ST, Piazza JR, Urban EJ. 2017 Mixed Emotions Across Adulthood: When, Where, and Why? Curr Opin Behav Sci 15, 58-61. (doi:10.1016/j.cobeha.2017.05.007)

75. Harmon-Jones E, Peterson CK. 2009 Supine body position reduces neural response to anger evocation. Psychol Sci 20, 1209-1210. (doi:10.1111/j.1467-9280.2009.02416.x)

76. Wilson-Mendenhall CD, Barrett LF, Barsalou LW. 2015 Variety in emotional life: withincategory typicality of emotional experiences is associated with neural activity in large-scale brain networks. Soc Cogn Affect Neurosci 10, 62-71. (doi:10.1093/scan/nsu037)

77. Righart R, de Gelder B. 2006 Context influences early perceptual analysis of faces--an electrophysiological study. Cereb Cortex 16, 1249-1257. (doi:10.1093/cercor/bhj066)

78. Gendron M, Lindquist KA, Barsalou L, Barrett LF. 2012 Emotion Words Shape Emotion Percepts. Emotion 12, 314-325. (doi:10.1037/a0026007)

79. Satpute AB, Nook EC, Narayanan S, Shu J, Weber J, Ochsner KN. 2016 Emotions in "Black and White" or Shades of Gray? How We Think About Emotion Shapes Our Perception and Neural Representation of Emotion. Psychol Sci 27, 1428-1442. (doi:10.1177/0956797616661555)

80. Oh VYS. 2022 Torn Between Valences: Mixed Emotions Predict Poorer Psychological Well-Being and Job Burnout. J Happiness Stud 23, 2171-2200. (doi:10.1007/s10902-021-00493-z) 81. Barca L, Marchis MD. 2018 The case of Sofia: an example of the dynamic properties of the therapeutic relationship. (doi:10.31219/osf.io/s3m6p) 
82. Torregrossa LJ, Snodgress MA, Hong SJ, Nichols HS, Glerean E, Nummenmaa L, Park S. 2019 Anomalous Bodily Maps of Emotions in Schizophrenia. Schizophr Bull 45, 1060-1067. (doi:10.1093/schbul/sby179)

83. Lloyd CS, Stafford E, McKinnon MC, Rabellino D, D'Andrea W, Densmore M, Thome J, Neufeld RWJ, Lanius RA. 2021 Mapping alexithymia: Level of emotional awareness differentiates emotion-specific somatosensory maps. Child Abuse \& Neglect 113, 104919.

(doi:10.1016/j.chiabu.2020.104919)

84. Kashdan TB, Steger MF. 2006 Expanding the topography of social anxiety. An experiencesampling assessment of positive emotions, positive events, and emotion suppression. Psychol Sci 17, 120-128. (doi:10.1111/j.1467-9280.2006.01674.x)

85. Niedenthal PM. 2007 Embodying emotion. Science 316, 1002-1005. (doi:10.1126/science.1136930)

86. Pezzulo G, Barca L, Friston KJ. 2015 Active inference and cognitive-emotional interactions in the brain. Behav Brain Sci 38, e85. (doi:10.1017/S0140525X14001009)

87. Vergallito A, Petilli MA, Cattaneo L, Marelli M. 2019 Somatic and visceral effects of word valence, arousal and concreteness in a continuum lexical space. Sci Rep 9, 20254.

(doi:10.1038/s41598-019-56382-2)

88. Pezzulo G, Iodice P, Barca L, Chausse P, Monceau S, Mermillod M. 2018 Increased heart rate after exercise facilitates the processing of fearful but not disgusted faces. Sci Rep 8, 398 . (doi:10.1038/s41598-017-18761-5)

89. Yu AANC, Iodice P, Pezzulo G, Barca L. 2020 Bodily Information and Top-Down Affective Priming Jointly Affect the Processing of Fearful Faces. (doi:10.31234/osf.io/h76a9)

90. Barrett LF, Simmons WK. 2015 Interoceptive predictions in the brain. Nat Rev Neurosci 16, 419-429. (doi:10.1038/nrn3950)

91. Seth AK. 2013 Interoceptive inference, emotion, and the embodied self. Trends in Cognitive Sciences 17, 565-573. (doi:10.1016/j.tics.2013.09.007)

92. Tschantz A, Barca L, Maisto D, Buckley CL, Seth AK, Pezzulo G. 2021 Simulating homeostatic, allostatic and goal-directed forms of interoceptive control using Active Inference. , 2021.02.16.431365. (doi:10.1101/2021.02.16.431365)

93. Pezzulo G, Rigoli F, Friston KJ. 2015 Active Inference, homeostatic regulation and adaptive behavioural control. Progress in Neurobiology 136, 17-35.

94. Iodice P, Porciello G, Bufalari I, Barca L, Pezzulo G. 2019 An interoceptive illusion of effort induced by false heart-rate feedback. PNAS 116, 13897-13902.

(doi:10.1073/pnas.1821032116)

95. Smith R, Kuplicki R, Feinstein J, Forthman KL, Stewart JL, Paulus MP, Investigators T 1000, Khalsa SS. 2020 A Bayesian computational model reveals a failure to adapt interoceptive precision estimates across depression, anxiety, eating, and substance use disorders. medRxiv, 2020.06.03.20121343. (doi:10.1101/2020.06.03.20121343)

96. Widen SC, Russell JA. 2003 A closer look at preschoolers' freely produced labels for facial expressions. Dev Psychol 39, 114-128. (doi:10.1037//0012-1649.39.1.114)

97. Widen SC, Russell JA. 2008 Children acquire emotion categories gradually. Cognitive Development 23, 291-312. (doi:10.1016/j.cogdev.2008.01.002)

98. Ruba AL, Meltzoff AN, Repacholi BM. 2020 Superordinate categorization of negative facial expressions in infancy: The influence of labels. Dev Psychol 56, 671-685. (doi:10.1037/dev0000892)

99. Bradley MM, Lang PJ. 1999 Affective norms for English words (ANEW): Instruction manual and affective ratings.

100. Coltheart M, Davelaar E, Jonasson JT, Besner D. 1977 Access to the internal lexicon. S. Dornick (ed.) Attention and performance, volume VI, 535-556.

101. Davis CJ, Perea M. 2005 BuscaPalabras: A program for deriving orthographic and phonological neighborhood statistics and other psycholinguistic indices in Spanish. Behavior 
Research Methods 37, 665-671.

102. Bertinetto PM, Burani C, Laudanna A, Marconi L, Ratti D, Rolando C, Thornton AM. 2005 Colfis (corpus e lessico di frequenza dell'italiano scritto). Available on http://www. istc. cnr. it/material/database , 67-73. 


\section{Appendix 1A. List of experimental stimuli}

The stimuli used in the mouse-tracking task are adjectives taken from a database of Italian words with affective norms [40], adapted from the Affective Norms for English Words (ANEW; [99]). The final list of 24 adjectives has been selected based on mean valence and arousal ratings, in order to have 12 positively valenced words (1-6 with high arousal, 7-12 with low arousal) and 12 negatively valenced words (13-18 with high arousal, 19-24 with low arousal). Psycholinguistic indexes have also been taken into account, in order to exclude words rated as too familiar/unfamiliar, too frequent/unfrequent.

Table 1 - List of 24 Italian words with English translation and mean+sd ratings of valence, arousal and familiarity. M_Val: Mean valence ratings, with 1 being very unpleasant and 9 very pleasant; M_Aro: Mean arousal ratings, with 1 being very calm and 9 very aroused; M_Fam: Mean familiarity ratings, with 1 being unfamiliar and 9 highly familiar. Standard deviation are also provided.

\begin{tabular}{|r|l|l|c|c|c|c|c|c|}
\hline & Eng_Word & Ita_Word & M_Val & SD_Val & M_Aro & SD_Aro & M_Fam & SD_Fam \\
\hline 1 & brave & coraggioso & 7.70 & 1.07 & 7.12 & 1.56 & 6.65 & 2.03 \\
\hline 2 & proud & orgoglioso & 6.03 & 2.66 & 6.18 & 2.38 & 7.25 & 1.33 \\
\hline 3 & astonished & stupito & 6.03 & 1.85 & 6.06 & 2.09 & 6.85 & 1.35 \\
\hline 4 & nice & simpatico & 8.42 & 0.94 & 6.18 & 2.60 & 7.90 & 1.29 \\
\hline 5 & social & socievole & 7.45 & 1.48 & 5.94 & 2.34 & 7.40 & 1.47 \\
\hline 6 & satisfied & soddisfatto & 8.44 & 0.88 & 6.69 & 2.57 & 7.15 & 2.11 \\
\hline 7 & respectful & rispettoso & 7.67 & 1.22 & 4.79 & 2.71 & 7.05 & 1.54 \\
\hline 8 & reserved & riservato & 6.20 & 1.76 & 4.40 & 1.85 & 6.80 & 1.28 \\
\hline 9 & relaxed & rilassato & 8.38 & 1.04 & 3.50 & 2.83 & 6.90 & 1.68 \\
\hline 10 & untroubled & sereno & 8.09 & 1.20 & 4.83 & 3.02 & 7.05 & 1.99 \\
\hline 11 & nonchalant & disinvolto & 6.80 & 1.47 & 4.80 & 2.08 & 6.80 & 1.51 \\
\hline 12 & carefree & spensierato & 7.53 & 2.06 & 4.09 & 2.82 & 5.80 & 2.07 \\
\hline 13 & anxious & ansioso & 2.16 & 1.22 & 7.25 & 1.77 & 7.35 & 1.63 \\
\hline 14 & disgusted & disgustato & 2.09 & 1.20 & 6.40 & 1.50 & 7.10 & 1.55 \\
\hline 15 & terrified & terrorizzato & 1.70 & 1.00 & 7.66 & 1.52 & 6.05 & 1.82 \\
\hline 16 & angry & arrabbiato & 2.25 & 1.39 & 7.00 & 1.95 & 7.65 & 1.39 \\
\hline 17 & scared & spaventato & 2.47 & 1.54 & 6.53 & 2.42 & 6.40 & 1.70 \\
\hline 18 & despairing & disperato & 2.48 & 1.52 & 6.06 & 2.18 & 6.75 & 1.94 \\
\hline 19 & shy & schivo & 3.88 & 1.24 & 3.48 & 1.37 & 6.10 & 1.52 \\
\hline 20 & detached & distaccato & 3.27 & 1.95 & 5.20 & 1.91 & 6.60 & 1.73 \\
\hline 21 & listless & apatico & 2.48 & 1.56 & 5.45 & 1.99 & 5.85 & 2.56 \\
\hline 22 & timid & timido & 3.94 & 1.86 & 4.89 & 2.26 & 7.70 & 1.53 \\
\hline 23 & indifferent & indifferente & 3.68 & 2.09 & 4.87 & 2.05 & 5.85 & 2.46 \\
\hline 24 & lonely & solitario & 3.45 & 1.92 & 5.33 & 1.59 & 6.55 & 1.57 \\
\hline
\end{tabular}


Table 2 Psycholinguistic indexes: word frequency and ortographic neighbours. FreqColfis and Ln_Colfis columns: The written frequency of use for each Italian word according to the CoLFIS corpus (Bertinetto et al., 2005) and the natural logarithm of this frequency plus one. N_OrtNeig: The number of orthographic neighbors for each Italian word. Two words are considered orthographic neighbors when they share all letters (in the same position) except one [100,101]. MeanFreq_Neig: The mean written frequency of use of the orthographic neighbors for each Italian word according to the CoLFIS corpus [102]. We obtained this and the previous indexes by using an algorithm available at the following site from Padua University: http://dpss.psy.unipd.it/claudio/vicini2.php.

$\begin{array}{lllcccc} & \text { Eng_Word } & \text { Ita_Word } & \text { FreqColfis } & \text { Ln_Colfis } & \text { N_OrtNeig } & \text { MeanFreq_Neig } \\ 1 & \text { brave } & \text { coraggioso } & 80 & 4.39 & 3 & 5.013717 \\ 2 & \text { proud } & \text { orgoglioso } & 76 & 4.34 & 3 & 4.491443 \\ 3 & \text { astonished } & \text { stupito } & 52 & 3.97 & 4 & 5.405424 \\ 4 & \text { nice } & \text { simpatico } & 132 & 4.89 & 2 & 9.40074 \\ 5 & \text { social } & \text { socievole } & 2 & 1.10 & 1 & 0.313357 \\ 6 & \text { satisfied } & \text { soddisfatto } & 139 & 4.94 & 3 & 7.729473 \\ 7 & \text { respectful } & \text { rispettoso } & 30 & 3.43 & 4 & 1.801804 \\ 8 & \text { reserved } & \text { riservato } & 106 & 4.67 & 4 & 9.949095 \\ 9 & \text { relaxed } & \text { rilassato } & 31 & 3.47 & 6 & 1.566787 \\ 10 & \text { untroubled } & \text { sereno } & 138 & 4.93 & 4 & 9.714082 \\ 11 & \text { nonchalant } & \text { disinvolto } & 34 & 3.56 & 3 & 2.402407 \\ 12 & \text { carefree } & \text { spensierato } & 9 & 2.30 & 2 & 0.940072 \\ 13 & \text { anxious } & \text { ansioso } & 57 & 4.06 & 3 & 4.491457 \\ 14 & \text { disgusted } & \text { disgustato } & 12 & 2.56 & 2 & 1.25343 \\ 15 & \text { terrified } & \text { terrorizzato } & 45 & 3.83 & 4 & 2.663538 \\ 16 & \text { angry } & \text { arrabbiato } & 44 & 3.81 & 4 & 2.036823 \\ 17 & \text { scared } & \text { spaventato } & 62 & 4.14 & 4 & 4.700354 \\ 18 & \text { despairing } & \text { disperato } & 165 & 5.11 & 4 & 9.949091 \\ 19 & \text { shy } & \text { schivo } & 9 & 2.30 & 3 & 6.267151 \\ 20 & \text { detached } & \text { distaccato } & 23 & 3.18 & 4 & 0.861733 \\ 21 & \text { listless } & \text { apatico } & 2 & 1.10 & 1 & 0.626715 \\ 22 & \text { timid } & \text { timido } & 83 & 4.43 & 3 & 3.86474 \\ 23 & \text { indifferent } & \text { indifferente } & 82 & 4.42 & 1 & 9.08737 \\ 24 & \text { lonely } & \text { solitario } & 78 & 4.37 & 2 & 4.54368\end{array}$


Table 3. Mean and standard deviation of written frequency (natural logarithm) from the CoLFIS corpus (Bertinetto et al., 2005)

\begin{tabular}{|l|c|c|}
\hline & Mean & $\begin{array}{c}\text { Standard } \\
\text { Deviation }\end{array}$ \\
\hline Positive valence & 3.83 & 1.17 \\
\hline Negative valence & 3.61 & 1.13 \\
\hline High Arousal & 3.93 & 1.12 \\
\hline Low Arousal & 3.51 & 1.15 \\
\hline
\end{tabular}




\section{Appendix 1B. Mousetracking task: triplets composition}

The task consists of 288 trials, each showing a triplet of stimuli. The triplets of stimuli (middle, left, right) were divided in 6 conditions, named with a code of 3 letters corresponding to the position of each stimulus (middle, left, right). The letters can be a " $\mathrm{P}$ " for positively valenced stimuli or a "N" for negatively valenced stimuli. Therefore, the 6 conditions, each consisting of 48 trials, are the following: PPP; PPN/PNP; PNN; NNN; NNP/NPN; NPP.

Here is the list of the possible combinations of target-responses in an explicit form, naming "target" the stimulus in the middle and "responses" those on the left and right:

1) Positive target and positive responses;

2) Positive target and one response positive + one negative response, counterbalancing left/right position;

3) Positive target and negative responses;

4) Negative target and negative responses;

5) Negative target and one response negative + one positive response, counterbalancing left/right position;

6) Negative target and positive responses.

An extra code of 3 letters specifies the arousal of the stimuli (" $\mathrm{H}$ " for high, " $\mathrm{L}$ " for low). However, the arousal of the words is not controlled and counterbalanced as well as the valence. In fact, the valence has been carefully controlled so that there was the same number of trials (48) for all the different combinations of triplets (the cited 6 conditions). Instead, in the case of arousal, we do not have the same number of trials for each condition, but it varies (HHH-39 trials, HHL-33, HLH-40, HLL-32; LHH-33, LHL-39, LLH33, LLL-39).

In general, the positions of the words used in the trials (288) have all been accurately manipulated so that in the final list all the words appeared in the 3 positions (middle, left, right) the same number of times (12 times). For example, the word "brave" appeared 12 times in the middle, 12 on the left, 12 on the right. At the same time, all the possible combinations of words occur in the trials, which means that each word (e.g. "brave") is paired with all the others. 


\section{Appendix 1C. Questionnaires scores}

Table 1 Questionnaires scores for each participant. Body Perception Questionnaire (BPQ, Awareness subscale). Positive and Negative Affect Schedule (PANAS). Beck Depression Inventory (BDI-II).

\begin{tabular}{|c|c|c|c|c|c|}
\hline Sub & Gender & $\begin{array}{c}\text { BPQ } \\
\text { t-score }\end{array}$ & $\begin{array}{l}\text { PANAS } \\
\text { Positive }\end{array}$ & $\begin{array}{l}\text { PANAS } \\
\text { Negative }\end{array}$ & BDI_II \\
\hline 1 & 1 & 60.9 & 17.00 & 15.00 & 9.00 \\
\hline 2 & 1 & 43.5 & 26.00 & 16.00 & 11.00 \\
\hline 3 & 1 & 60.2 & 36.00 & 13.00 & 3.00 \\
\hline 4 & 2 & 61.7 & 38.00 & 22.00 & 16.00 \\
\hline 5 & 2 & 57.8 & 15.00 & 10.00 & 0.00 \\
\hline 6 & 2 & 57.5 & 15.00 & 20.00 & 8.00 \\
\hline 7 & 1 & 60.6 & 31.00 & 22.00 & 14.00 \\
\hline 8 & 2 & 56.4 & 23.00 & 12.00 & 0.00 \\
\hline 9 & 1 & 49.6 & 25.00 & 10.00 & 3.00 \\
\hline 10 & 1 & 47.9 & 32.00 & 16.00 & 3.00 \\
\hline 11 & 2 & 65.7 & 23.00 & 15.00 & 8.00 \\
\hline 12 & 2 & 40.4 & 23.00 & 11.00 & 15.00 \\
\hline 13 & 1 & 52.3 & 31.00 & 13.00 & 1.00 \\
\hline 14 & 2 & 58.7 & 18.00 & 24.00 & 18.00 \\
\hline 15 & 2 & 65.2 & 30.00 & 23.00 & 9.00 \\
\hline 16 & 1 & 51.2 & 35.00 & 11.00 & 4.00 \\
\hline 17 & 2 & 53.8 & 16.00 & 11.00 & 7.00 \\
\hline 18 & 2 & 52.3 & 34.00 & 21.00 & 2.00 \\
\hline 19 & 2 & 61.1 & 33.00 & 26.00 & 22.00 \\
\hline 20 & 1 & 59.3 & 33.00 & 12.00 & 8.00 \\
\hline 21 & 1 & 49.2 & 15.00 & 11.00 & 8.00 \\
\hline 22 & 2 & 45.8 & 21.00 & 11.00 & 11.00 \\
\hline 23 & 2 & 74.1 & 37.00 & 24.00 & 12.00 \\
\hline 24 & 1 & 55.1 & 37.00 & 12.00 & 2.00 \\
\hline 25 & 1 & 62.3 & 23.00 & 16.00 & 12.00 \\
\hline 26 & 1 & 49.6 & 32.00 & 12.00 & 2.00 \\
\hline 27 & 2 & 52 & 28.00 & 13.00 & 2.00 \\
\hline 28 & 1 & 59.3 & 22.00 & 24.00 & 10.00 \\
\hline 29 & 2 & 74.1 & 36.00 & 22.00 & 15.00 \\
\hline 30 & 1 & 48.7 & 28.00 & 24.00 & 11.00 \\
\hline
\end{tabular}


Table 2. MAIA-2 scores across the 8 subscales (Multidimensional Assessment of Interoceptive Awareness. Version 2). Please note that we used a shorter version (5 steps) with a correction due to Mehling to compare with a 6-step scale.

\begin{tabular}{|c|cccccccc|}
\hline Sub & Noticing & $\begin{array}{c}\text { Not- } \\
\text { Distracting }\end{array}$ & $\begin{array}{c}\text { Not- } \\
\text { Worrying }\end{array}$ & $\begin{array}{c}\text { Attention } \\
\text { Regulation }\end{array}$ & $\begin{array}{c}\text { Emotional } \\
\text { Awareness }\end{array}$ & $\begin{array}{c}\text { Self- } \\
\text { Regulation }\end{array}$ & $\begin{array}{c}\text { Body } \\
\text { Listening }\end{array}$ & Trusting \\
\hline 1 & 3.3 & 2 & 3.12 & 2.057143 & 1.92 & 2.4 & 2.4 & 2.8 \\
& & & & & & & & \\
2 & 2.4 & 3 & 2.64 & 2.4 & 3.12 & 3.3 & 1.6 & 2.8 \\
3 & 3.3 & 3.2 & 1.44 & 2.571429 & 4.08 & 1.8 & 2.8 & 4 \\
4 & 2.1 & 3.2 & 0.72 & 1.714286 & 2.88 & 0.6 & 2 & 1.6 \\
5 & 2.1 & 4.2 & 0.24 & 2.914286 & 0 & 0.6 & 0 & 3.2 \\
6 & 3.6 & 2.4 & 0.72 & 1.885714 & 4.08 & 1.2 & 2 & 2.4 \\
7 & 3.6 & 2.8 & 1.2 & 3.085714 & 3.84 & 3.6 & 3.2 & 2.8 \\
8 & 3 & 2 & 1.92 & 1.371429 & 2.4 & 2.1 & 2 & 3.2 \\
9 & 1.8 & 2.4 & 2.64 & 1.542857 & 0.48 & 0.3 & 0.8 & 2.4 \\
10 & 3 & 2.4 & 2.64 & 2.4 & 2.88 & 3 & 2.8 & 3.2 \\
11 & 3.9 & 2.4 & 1.92 & 2.742857 & 3.6 & 2.4 & 3.2 & 4 \\
12 & 3 & 2 & 2.16 & 1.714286 & 3.6 & 1.2 & 2.4 & 1.6 \\
13 & 2.4 & 2.4 & 1.92 & 3.428571 & 2.64 & 3.6 & 3.2 & 4.4 \\
14 & 3.3 & 2.8 & 0.48 & 1.714286 & 4.32 & 2.7 & 2.8 & 2 \\
15 & 3.6 & 1.8 & 1.2 & 2.4 & 3.12 & 3.6 & 3.2 & 3.6 \\
16 & 1.8 & 2 & 3.12 & 1.885714 & 2.64 & 1.5 & 1.6 & 3.2 \\
17 & 0.9 & 3.4 & 2.64 & 0.685714 & 0.72 & 2.1 & 1.2 & 0 \\
18 & 3.3 & 2.6 & 2.16 & 2.742857 & 3.84 & 3 & 2.4 & 3.6 \\
19 & 3.9 & 1 & 1.68 & 3.428571 & 3.84 & 3.3 & 3.6 & 4 \\
20 & 3.6 & 3.4 & 2.4 & 3.771429 & 3.36 & 3.3 & 3.6 & 4.4 \\
21 & 3.3 & 2.8 & 2.4 & 2.4 & 3.6 & 1.5 & 2 & 3.2 \\
22 & 2.1 & 2.4 & 3.6 & 1.885714 & 1.44 & 1.8 & 2.4 & 1.6 \\
23 & 3.6 & 2.8 & 1.2 & 2.914286 & 4.56 & 1.8 & 4.8 & 2.4 \\
24 & 1.5 & 2.4 & 3.12 & 3.085714 & 3.84 & 4.8 & 4 & 4.4 \\
25 & 3.3 & 1 & 0.72 & 1.714286 & 3.84 & 1.8 & 3.2 & 3.2 \\
\hline
\end{tabular}




\begin{tabular}{|l|cccccccc|}
\hline 26 & 2.4 & 2.2 & 2.4 & 2.742857 & 2.88 & 2.4 & 2.4 & 3.2 \\
27 & 1.8 & 2.8 & 1.44 & 1.885714 & 2.88 & 1.8 & 2.8 & 2.8 \\
28 & 3.3 & 1.6 & 1.2 & 1.714286 & 3.6 & 2.1 & 2 & 2.4 \\
29 & 3 & 2.6 & 2.4 & 3.428571 & 3.6 & 4.2 & 3.6 & 3.2 \\
30 & 3.6 & 2.8 & 2.16 & 2.571429 & 3.36 & 2.7 & 3.2 & 3.2 \\
\hline
\end{tabular}




\section{Appendix 1D. HRV scores}

\begin{tabular}{|c|cccccc|}
\hline & \multicolumn{2}{|c|}{ TIME DOMAIN } & \multicolumn{4}{c|}{ FREQUENCY DOMAIN (AR/ms2) } \\
\hline Sub & $\begin{array}{c}\text { MEAN HR } \\
\text { (beats/min) }\end{array}$ & $\begin{array}{c}\text { RMSSD } \\
\text { (ms) }\end{array}$ & VLF & LF & HF & LF/HF \\
\hline 1 & 79.141 & 41.154 & 107.99 & 3231.5 & 654.84 & 4.9348 \\
& & & & & & \\
2 & 76.103 & 19.768 & 45.749 & 189.85 & 169.02 & 1.1232 \\
3 & 62.867 & 88.4 & 221.73 & 2146.7 & 2639.4 & 0.81334 \\
4 & 77.968 & 60.598 & 313.4 & 1574.6 & 1918.5 & 0.8207 \\
5 & 81.911 & 41.59 & 89.605 & 1091.6 & 744.16 & 1.4669 \\
6 & 71.724 & 29.115 & 197.08 & 856.65 & 312.16 & 2.7443 \\
7 & 60.188 & 24.293 & 172.52 & 1285.6 & 128.16 & 10.032 \\
8 & 82.346 & 36.973 & 94.056 & 430.05 & 535.94 & 0.80243 \\
9 & 65.703 & 41.548 & 105.77 & 802.59 & 639.41 & 1.2552 \\
10 & 73.403 & 29.62 & 149.18 & 831.34 & 360.98 & 2.303 \\
11 & 66.131 & 75.231 & 181.21 & 1839.3 & 2754.8 & 0.66769 \\
12 & 93.069 & 12.244 & 23.286 & 112.2 & 62.299 & 1.801 \\
13 & 63.507 & 27.523 & 57.65 & 902.53 & 216.77 & 4.1635 \\
14 & 76.669 & 32.411 & 121.42 & 652.7 & 591.06 & 1.1043 \\
15 & 78.204 & 45.174 & 44.563 & 388.62 & 1005.4 & 0.38652 \\
16 & 72.35 & 29.948 & 163.2 & 991.93 & 298.13 & 3.3272 \\
17 & 90.753 & 18.256 & 39.415 & 215.86 & 140.45 & 1.5369 \\
18 & 86.268 & 35.5 & 130.6 & 815.58 & 573.79 & 1.4214 \\
19 & 76.625 & 37.168 & 95.594 & 604.17 & 896.41 & 0.67398 \\
20 & 65.164 & 33.653 & 68.457 & 707.47 & 493.43 & 1.4338 \\
21 & 74.023 & 61.786 & 265.56 & 2137.5 & 1472.7 & 1.4514 \\
22 & 71.611 & 42.516 & 193.67 & 606.94 & 868.73 & 0.69866 \\
23 & 77.845 & 28.313 & 75.652 & 518.44 & 409.3 & 1.2666 \\
24 & 63.063 & 36.579 & 156.26 & 953.77 & 479.98 & 1.9871 \\
25 & 71.56 & 56.068 & 76.17 & 646.46 & 1444.7 & 0.44748 \\
26 & 89.921 & 36.69 & 502.13 & 2168.6 & 599.28 & 3.6188 \\
27 & 57.795 & 109.97 & 278.55 & 1808.1 & 3888.9 & 0.46493 \\
28 & 64.78 & 117.48 & 304.27 & 2463.7 & 5694 & 0.43268 \\
29 & 70.596 & 31.484 & 42.606 & 720.45 & 333.64 & 2.1593 \\
30 & 75.483 & 30.34 & 63.551 & 335.08 & 300.3 & 1.1158
\end{tabular}




\section{Appendix 1E.}

\section{Power Analysys}

> val.lmer=lmer $(\mathrm{RT} \sim$ TargetValence * CongruencyTargetValence $+(1 \mid$ subject $)+(1 \mid$ trial $)$, data=prova 1$)$

$>\mathrm{R} 2<-$ r.squaredLR(val.lmer)

$>\mathrm{R} 2$

[1] 0.2192279

attr(,"adj.r.squared")

[1] 0.219228

$>\mathrm{f} 2=(\mathrm{R} 2 /(1-\mathrm{R} 2))$

$>\mathrm{f} 2$

[1] 0.2807835

attr(,"adj.r.squared")

[1] 0.219228

$>$ pwr.f2.test $(\mathrm{u}=4, \mathrm{v}=30, \mathrm{f} 2=.28$, sig.level $=.05)$

Multiple regression power calculation

$\mathrm{u}=4$

$\mathrm{v}=30$

$\mathrm{f} 2=0.28$

sig.level $=0.05$

power $=0.6282272$ 


\section{Outputs of LMMS}

Table 1. Output of LMMs on response time considering the valence dimension. reporting the estimates of the model. confidence interval (CI) and p-values (calculated from an $\mathrm{F}$ test and test based on Sattethwaite's approximation).

\begin{tabular}{|c|c|c|c|}
\hline \multirow[b]{2}{*}{ Predictors } & \multicolumn{3}{|c|}{ RT } \\
\hline & Estimates & $C I$ & $p$ \\
\hline (Intercept) & 2033.56 & $1956.36-2110.76$ & $<0.001$ \\
\hline TargetValencepos & 17.74 & $-11.90-47.38$ & 0.241 \\
\hline Congruency TargetValenceCong1 & -195.45 & $-224.86--166.03$ & $<0.001$ \\
\hline Congruency TargetValenceCong2 & -130.25 & $-159.67--100.82$ & $<0.001$ \\
\hline TargetValencepos:CongruencyTargetValenceCong1 & 17.04 & $-24.59-58.68$ & 0.422 \\
\hline TargetValencepos:CongruencyTargetValenceCong2 & 10.85 & $-30.80-52.50$ & 0.610 \\
\hline \multicolumn{4}{|l|}{ Random Effects } \\
\hline$\sigma^{2}$ & \multicolumn{3}{|l|}{155246.45} \\
\hline$\tau_{00 \text { trial }}$ & \multicolumn{3}{|l|}{362.93} \\
\hline$\tau_{00}$ subject & \multicolumn{3}{|l|}{43048.12} \\
\hline $\mathrm{ICC}_{\text {trial }}$ & \multicolumn{3}{|l|}{0.00} \\
\hline $\mathrm{ICC}_{\text {subject }}$ & \multicolumn{3}{|l|}{0.22} \\
\hline Observations & \multicolumn{3}{|l|}{8328} \\
\hline Marginal $\mathrm{R}^{2} /$ Conditional $\mathrm{R}^{2}$ & \multicolumn{3}{|c|}{$0.030 / 0.242$} \\
\hline
\end{tabular}


Table 2. Outpunt of LMMs on response time considering the arousal dimension. reporting the estimates of the model. confidence interval $(\mathrm{CI})$ and $\mathrm{p}$-values (calculated from an $\mathrm{F}$ test and test based on Sattethwaite's approximation).

\begin{tabular}{|c|c|c|c|}
\hline \multirow[b]{2}{*}{ Predictors } & \multicolumn{3}{|c|}{ RT } \\
\hline & Estimates & $C I$ & $p$ \\
\hline (Intercept) & 1949.51 & $1870.98-2028.04$ & $<0.001$ \\
\hline TargetArousallow & 127.57 & $91.33-163.81$ & $<0.001$ \\
\hline CongruencyTargetArousalCong1 & -13.29 & $-44.00-17.42$ & 0.396 \\
\hline CongruencyTargetArousalCong2 & 5.38 & $-29.20-39.97$ & 0.760 \\
\hline TargetArousallow:CongruencyTargetArousalCong1 & -171.73 & $-215.23--128.23$ & $<0.001$ \\
\hline TargetArousallow:CongruencyTargetArousalCong2 & -198.60 & $-247.49--149.71$ & $<0.001$ \\
\hline \multicolumn{4}{|l|}{ Random Effects } \\
\hline$\sigma^{2}$ & \multicolumn{3}{|l|}{158098.69} \\
\hline$\tau_{00 \text { trial }}$ & \multicolumn{3}{|l|}{600.78} \\
\hline$\tau_{00 \text { subject }}$ & \multicolumn{3}{|l|}{42935.88} \\
\hline $\mathrm{ICC}_{\text {trial }}$ & \multicolumn{3}{|l|}{0.00} \\
\hline $\mathrm{ICC}_{\text {subject }}$ & \multicolumn{3}{|l|}{0.21} \\
\hline Observations & \multicolumn{3}{|l|}{8328} \\
\hline Marginal $\mathrm{R}^{2} /$ Conditional $\mathrm{R}^{2}$ & \multicolumn{3}{|c|}{$0.015 / 0.228$} \\
\hline
\end{tabular}




\section{Appendix 1F. Supplementary Figures}
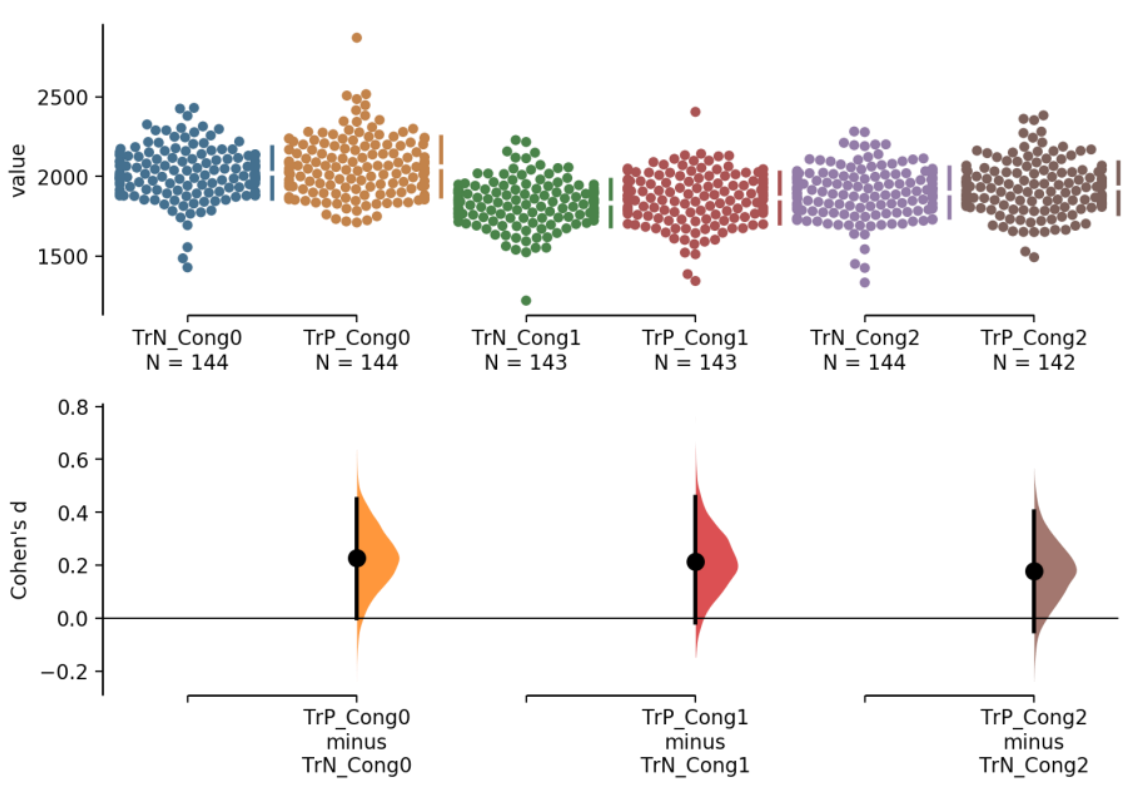

Figure S1. Cumming estimation plot on Response time data as a function of emotional Valence (comparison between target with positive and negative valence). The Cohen's $\mathrm{d}$ for 3 comparisons are shown in the above Cumming estimation plot. The raw data is plotted on the upper axes; each mean difference is plotted on the lower axes as a bootstrap sampling distribution. Mean differences are depicted as dots; $95 \%$ confidence intervals are indicated by the ends of the vertical error bars. $\operatorname{TrN}=$ target with negative valence. $\operatorname{TrP}=$ Target with positive valence. Cong $0=$ no congruency with responses. Cong $1=$ valence congruency with one response. Cong 2 = valence congruency with both responses.
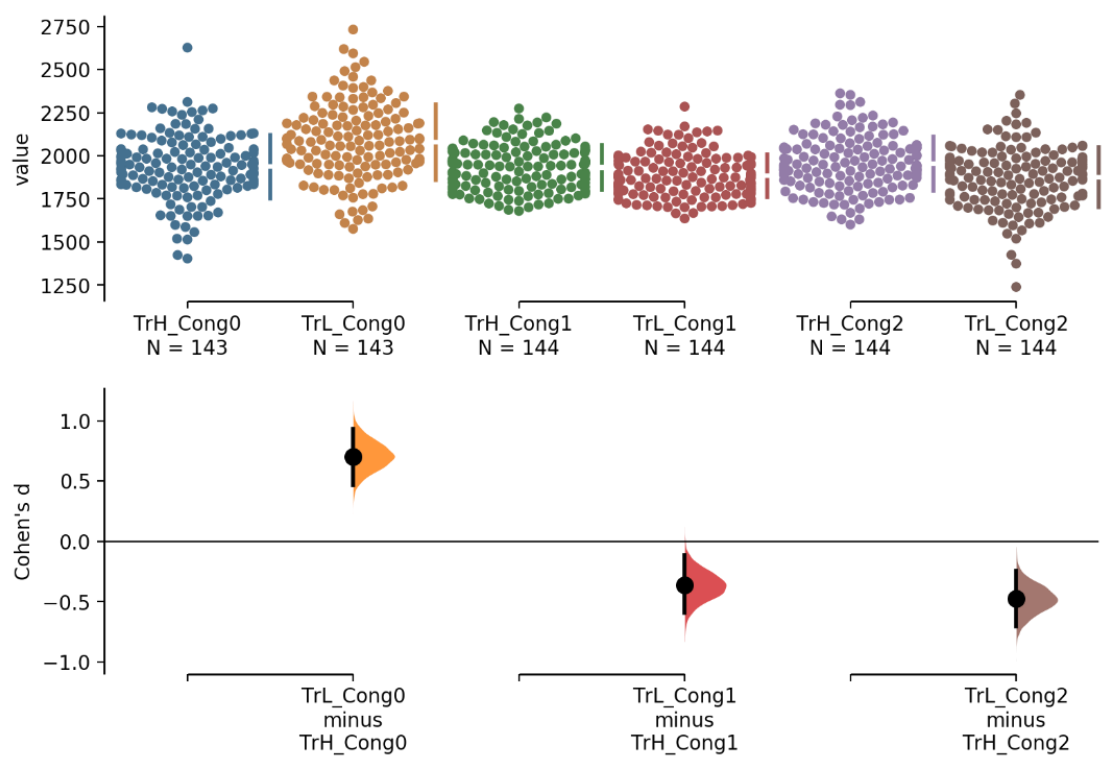

Figure S2. Cumming estimation plot on Response time data as a function of emotional Arousal (comparison between target with high and low Arousal). The Cohen's d for 3 comparisons are shown in the above Cumming estimation plot. The raw data is plotted on 
the upper axes; each mean difference is plotted on the lower axes as a bootstrap sampling distribution. Mean differences are depicted as dots; $95 \%$ confidence intervals are indicated by the ends of the vertical error bars. $\mathrm{TrH}=$ target with high arousal. $\operatorname{TrL}=$ Target with low arousal. Cong $0=$ no congruency with responses. Cong1= arousal congruency with one response. Cong 2 = arousal congruency with both responses.

\section{Appendix 1G. Inclusion criteria}

Participants included in the group High Negativity: 14. 15. 19. 23. 28.30

Participants included in the group Low Positivity: 5. 9. 12. 17. 21.22

Participants included in the group High HRV: 2. 10. 26. 271

Participants included in the group Low HRV:. 11. 16 